\title{
Consensus for Quantum Networks: Symmetry From Gossip Interactions
}

\author{
Luca Mazzarella, Alain Sarlette, and Francesco Ticozzi
}

\begin{abstract}
This paper extends the consensus framework, widely studied in the literature on distributed computing and control algorithms, to networks of quantum systems. We define consensus situations on the basis of invariance and symmetry properties, finding four different generalizations of classical consensus states. This new viewpoint can be directly used to study consensus for probability distributions, as these can be seen as a particular case of quantum statistical states: in this light, our analysis is also relevant for classical problems. We then extend the gossip consensus algorithm to the quantum setting and prove it converges to symmetric states while preserving the expectation of permutationinvariant global observables. Applications of the framework and the algorithms to estimation and control problems on quantum networks are discussed.
\end{abstract}

Index Terms-Consensus, distributed algorithms, Markov processes, quantum information and control.

\section{INTRODUCTION}

A MONG the recent trends in control and systems theory, the field of distributed control, estimation and optimization on networks has stimulated an impressive amount of research. A basic task for distributed information processing is reaching consensus on some common control objective, shared value or slack variable. The present paper extends this wellstudied consensus problem (see, e.g., [1], [2]) to networks of quantum systems, a special case of which would be classical probability distributions. We therefore try to make the main message accessible to non-quantum experts (see end of the Introduction).

Exploring the links between information processing tasks and stochastic dynamics on networks has recently opened new research directions towards "distributed" quantum information applications. In essence, these involve an interplay between symmetry, locality constraints, and engineered dissipation. These are the key ingredients in many quantum information applications, among which noise protection and dynamical error-correction [3]-[6], open-system quantum sim-

Manuscript received March 17, 2013; revised September 13, 2013 and February 18, 2014; accepted June 22, 2014. Date of publication July 8, 2014; date of current version December 22, 2014. This work was supported in part by a University of Padua QFUTURE grant. Recommended by Associate Editor C. Altafini.

L. Mazzarella is with the Dipartimento di Ingegneria dell'Informazione, Università di Padova, 35131 Padua, Italy (e-mail: mazzarella@ dei.unipd.it).

A. Sarlette is with SYSTeMS, Ghent University, 9052 Gent, Belgium (e-mail: alain.sarlette@ugent.be).

F. Ticozzi is with the Dipartimento di Ingegneria dell'Informazione, Università di Padova, 35131 Padova, Italy, and also with the Department of Physics and Astronomy, Dartmouth College, Hanover, NH 03755 USA (e-mail: ticozzi@dei.unipd.it).

Color versions of one or more of the figures in this paper are available online at http://ieeexplore.ieee.org.

Digital Object Identifier 10.1109/TAC.2014.2336351 ulators [7], [8] and quantum computers [9], entanglement generation through stabilizing dissipative dynamics [10], [11] as well as most tasks in the stabilization of open multipartite quantum systems [12]-[14].

In this spirit, we here develop a framework for quantum consensus: we identify and characterize a hierarchy of quantum consensus situations and study how these can be reached by suitable dissipative quantum dynamics, while preserving some global information on the network state. Our work offers not only a formal generalization of the well-known classical consensus problem, but also a potentially new viewpoint on a number of issues in quantum information. More specifically, it ties the structure of symmetric states and correlations [15], [16] to their potential generation via locality constrained resources, similarly in spirit to what has been recently done by characterizing another relevant class of states, namely frustrationfree ground states of quasi-local Hamiltonians with dissipative generators [11]. In addition, the ideas and methods we present can be directly employed to symmetrize the state of a large system towards permutation-invariant statistics, guarantee effective sampling from large networks of quantum systems, achieve robust broadcast of information, or realize purification and cooling with limited resources. More details about three possible direct applications of our gossip-type algorithm are presented in the following sections.

An attempt to lift the consensus problem to the quantum domain has been presented in [17]. It is based on a "cone geometry" approach, viewing quantum Kraus maps as the noncommutative generalization of Markov chain transition mechanisms that model consensus algorithms. The authors show how Birkhoff's Theorem and Hilbert's projective metric lead to a general convergence result and contraction ratio estimation. However, by describing the dynamics of the whole system of interest as governed by a single Markov transition mechanism, this formulation does not account for subsystem structure or network connections in the quantum setting. It therefore defines consensus as asymptotic convergence to a scalar multiple of the identity: for quantum states this corresponds to a fully mixed, most uncertain state which is rarely the desired target for applications.

In the present paper, we approach quantum consensus from an "operational," multi-agent control perspective, starting from the basic classical ingredients: a network of subsystems, an interaction protocol with locality constraints, and a target consensus situation. As a first step, Section II provides four possible ways to generalize the concept of a consensus state to the quantum domain and explores their connections, establishing a hierarchy of quantum consensus definitions. Section III presents the quantum open-system dynamics and the locality notions that we employ to describe the interactions between the quantum "agents." Typical methods for interaction selection and timing 
are also introduced, in analogy with classical consensus. This provides all the ingredients needed to specify the general properties of an evolution that achieves quantum average consensus. The symmetry-based reformulations at each step provide an alternative interpretation of classical consensus, and in Section IV this leads us to a quantum generalization of the classical gossip algorithm. We prove its convergence to symmetric-state consensus while preserving the expectation of any permutation-invariant observable. We further show how the algorithm can be explicitly seen as a generalization of the classical one and the classical convergence results can be used to prove a weaker convergence property. The section concludes with an example of the gossip algorithm working on a fourqubit network. Section V presents two potential applications of the framework and method. In Section VI, we summarize the main results and provide an outlook on possible further developments and applications.

For the readers who do not have prior knowledge of quantum theory: a brief tutorial on quantum systems modeling is given in Appendix A, along with an overview of the notations and conventions we use. In essence, the paper can be read more or less verbatim with the translation table shown at the bottom of the page.

Correspondingly, the (adjoint) $\dagger$ symbol indicates the transpose-conjugate in matrix representation, and the tensor product $\otimes$ is associated to the Kronecker matrix product.

A quantum state is in general associated with a density operator $\rho$, that is represented with a positive-semidefinite, traceone Hermitian matrix. The eigenvalues of such a matrix can be interpreted as the probabilities of $n$ mutually-exclusive outcomes of a real random variable $x$. Consider a basis in which $\rho$ is diagonal: the Kronecker product of $m$ such matrices then is a diagonal $n^{m} \times n^{m}$ matrix representing the product of marginal distributions of $m$ measurements of the random variable $x$. The space of all joint probability distributions for $m$ subsystems is larger than the product of marginals, since it allows for arbitrary correlations, and it is represented by all diagonal trace-one positive $n^{m} \times n^{m}$ matrices (not necessarily Kronecker-factorizable). The quantum context adds to this picture the possibility to arbitrarily "rotate" the bases in which things are expressed, and hence consider non-diagonal matrices and "non-commutative probabilities" (see, e.g., [18], [19] for a formal introduction to this viewpoint). However, our results can be applied in particular to diagonal states, and can hence be directly translated to classical probability distributions.

\section{Consensus States}

A classical consensus state for a multipartite system is one in which the states of all the subsystems, often called agents, are the same (although not necessarily stationary). Since in general a quantum state for a multipartite system cannot be factorized into subsystem states (see Appendix A), the definition of consensus must be reconsidered. We here present an operational approach that leads to a hierarchy of definitions for what can be claimed to be "quantum consensus." The resources needed for discriminating between them are discussed in Appendix C.

\section{A. Defining Classical Consensus}

Consensus for classical systems is typically formulated along the following lines [1], [2]. Consider $m$ subsystems, each one associated to a state given by a configuration variable $x_{k} \in \mathbb{R}^{n}$. These subsystems evolve through bilateral interactions, according to some networking scheme and dynamics (see Section IV), and reach a consensus state if they converge to the set $\mathcal{C}=$ $\left\{\left(x_{1}, x_{2}, \ldots, x_{m}\right): x_{j}=x_{k} \forall j, k\right\}$. Furthermore, the agents are said to compute an average consensus if for given initial states $\left(x_{1}(0), x_{2}(0), \ldots, x_{m}(0)\right)$ they converge to the particular equilibrium $(\bar{x}, \bar{x}, \ldots, \bar{x}) \in \mathcal{C}$ where $\bar{x}=(1 / m) \sum_{i=1}^{m} x_{i}(0)$.

Alternatively, consensus can be characterized as invariance with respect to subsystem permutations. Let $\mathfrak{P}$ denote the set of all subsystem permutation operators, i.e., each $P_{\pi} \in \mathfrak{P}$ is associated to some permutation $\pi$ of the integers $1,2, \ldots m$ such that $P_{\pi}\left(x_{1}, x_{2}, \ldots, x_{m}\right)=\left(x_{\pi(1)}, x_{\pi(2)}, \ldots, x_{\pi(m)}\right)$ for any $x_{1}, x_{2}, \ldots, x_{m}$. Denoting the joint state of the $x_{k} \in \mathbb{R}^{n}$ by a vector $x \in \mathbb{R}^{m n}$, each $P_{\pi} \in \mathfrak{P}$ can be written as an $m n \times m n$ matrix resulting from the Kronecker product of some $m \times m$ permutation matrix with the $n \times n$ identity matrix. Then we can define consensus as

$$
\mathcal{C}=\left\{x \in \mathbb{R}^{m n}: P_{\pi} x=x \text { for all } P_{\pi} \in \mathfrak{B}\right\} .
$$

Obviously, checking $P_{\pi} x=x$ for all pairwise permutations $P_{\pi}$ is sufficient to guarantee $P_{\pi} x=x$ for all $P_{\pi} \in \mathfrak{P}$.

Average consensus algorithms represent a useful tool for many tasks in distributed control and computation because for each linear function $Q: \mathbb{R}^{m n} \rightarrow \mathbb{R}$ which is invariant under all subsystem permutations, there exists $q: \mathbb{R}^{n} \rightarrow \mathbb{R}$ such that $Q x=q \bar{x}$, where $\bar{x}$ is the single subsystem state at consensus. This means that the value of such linear functional $Q$ of a global, $m \cdot n$ dimensional variable, can be obtained locally by each agent once they have reached average consensus, by evaluating an $n$-dimensional functional $q$. The fact that all permutations can be obtained by concatenating pairwise permutations, already suggests that average consensus is computable with distributed algorithms.

\section{B. Quantum Consensus Definitions and Their Relationships}

Defining what a consensus situation ought to be in a quantum "network" is not a straightforward task. More than one definition may be appropriate depending on the type of symmetry we are seeking. Following the analogy with the classical case can help, but quantum measurement outcomes are intrinsically

\begin{tabular}{|ll|}
\hline $\mathcal{H}, n$-dimensional Hilbert space & $\rightarrow \mathbb{C}^{n}$ \\
$|x\rangle \in \mathcal{H}$ & $\rightarrow$ column vector, $x \in \mathbb{C}^{n}$ \\
$\langle x| \in \mathcal{H}^{\dagger}$ & $\rightarrow$ row vector, $x^{\dagger}$ \\
$X$, operator & $\rightarrow X$, complex matrix.
\end{tabular}


stochastic, so we must consider probabilistic consensus situations from the beginning. Let us explore different options by first discussing a simple case. We refer to Appendix A2 for a short introduction to the standard notation for composite quantum systems, qubits, and Pauli matrices as used in the following.

Example 1-When is a Quantum Network in Consensus?: Consider a multipartite quantum system composed of three qubits, with associated Hilbert space $\mathcal{H}^{3}=\mathbb{C}^{2} \otimes \mathbb{C}^{2} \otimes \mathbb{C}^{2}$ spanned by $2^{3}$ basis vectors denoted by $\{|a, b, c\rangle=|a\rangle \otimes|b\rangle \otimes$ $|c\rangle: a, b, c \in\{0,1\}\}$, and three observables of the form $\sigma^{(1)}=$ $\sigma_{z} \otimes I \otimes I, \sigma^{(2)}=I \otimes \sigma_{z} \otimes I, \sigma^{(3)}=I \otimes I \otimes \sigma_{z}$, where the Pauli matrix $\sigma_{z}=\operatorname{diag}(1,-1)$ with respect to the ordered basis $\{|0\rangle,|1\rangle\}$. These correspond to observables of the quantity associated to $\sigma_{z}$ for each of the subsystems, i.e., measuring $\sigma^{(3)}$ gives result +1 (resp. -1 ) if the third qubit is in state $|0\rangle$ (resp. $|1\rangle)$. It seems natural to say that the system is in consensus with respect to the expectation of $\sigma_{z}$ if

$$
\operatorname{Tr}\left(\rho \sigma^{(1)}\right)=\operatorname{Tr}\left(\rho \sigma^{(2)}\right)=\operatorname{Tr}\left(\rho \sigma^{(3)}\right) .
$$

The conditions for this to happen can be worked out explicitly in terms of the diagonal elements of the state $\rho$. In particular it is easy to check that all the following states satisfy (2):

$$
\begin{aligned}
\rho^{A} & =\frac{1}{8} I \otimes(|0\rangle+|1\rangle)(\langle 0|+\langle 1|) \otimes(|0\rangle+|1\rangle)(\langle 0|+\langle 1|) \\
\rho^{B} & =\frac{1}{2}(|0,0,1\rangle\langle 0,0,1|+| 1,1,0\rangle\langle 1,1,0|) \\
\rho^{C} & =\frac{1}{8} I \otimes I \otimes I \\
\rho^{D} & =\frac{1}{2}(|0,0,0\rangle\langle 0,0,0|+| 1,1,1\rangle\langle 1,1,1|) \\
\rho^{E} & =|0,0,0\rangle\langle 0,0,0| \\
\rho^{F} & \left.=\frac{1}{2}(|0,0,0\rangle+|1,1,1\rangle)(\rangle 0,0,0 \mid+\langle 1,1,1|\right) .
\end{aligned}
$$

All these states, except $\rho^{E}$, have $\operatorname{Tr}\left(\rho \sigma^{(i)}\right)=0$ for $i=1,2,3$. The states $\rho^{B}, \rho^{C}, \rho^{D}, \rho^{E}$ are diagonal in the canonical basis and hence can be interpreted as classical probabilities on the set $\{-1,+1\} \times\{-1,+1\} \times\{-1,+1\}$ of possible outcomes for the joint measurements of $\sigma^{(j)}, j=1,2,3$.

The requirement (2) can be strengthened by requesting it to hold when $\sigma_{z}$ is replaced by any observable $\sigma \in \mathfrak{H}\left(\mathbb{C}^{2}\right)$ in the definition of $\sigma^{(1)}, \sigma^{(2)}, \sigma^{(3)}$. This is equivalent to imposing that the reduced states for the three subsystems are the same. It is then easy to check that $\rho^{B}, \rho^{C}, \rho^{D}, \rho^{E}, \rho^{F}$ satisfy this requirement, while $\rho^{A}$ does not. In fact the reduced states for $\rho^{A}$ are

$$
\rho_{1}^{A}=\frac{1}{2} I, \quad \rho_{2}^{A}=\rho_{3}^{A}=\frac{1}{2}(|0\rangle+|1\rangle)(\langle 0|+\langle 1|) .
$$

In the light of (1), another potential definition of quantum consensus would require the state to be symmetric, i.e., invariant under any permutations of the subsystems. This choice can be motivated by the classical case, where the consensus state is indeed permutation invariant. Among the states defined in Example 1, only $\rho^{C}, \rho^{D}, \rho^{E}, \rho^{F}$ are permutation invariant.

Lastly, one might want subsystem agreement not only on the observable averages, but on each realization of a stochastic measurement (see Appendix A2); namely, that each projective measurement of the (commuting and hence compatible) observables $\sigma^{(1)}, \sigma^{(2)}, \sigma^{(3)}$ gives perfectly correlated results for the three subsystems. Thus, among all possible measurement results $\{-1,+1\}^{\times 3}$, one wants that only $(-1,-1,-1)$ and $(+1,+1,+1)$ have a nonzero probability to occur. ${ }^{1}$ The states $\rho^{A}, \rho^{B}$, and $\rho^{C}$ do not satisfy this definition of consensus; indeed, for these three states, the distribution of measurement results for qubit 1 is either independent $\left(\rho^{A}, \rho^{C}\right)$ or anticorrelated $\left(\rho^{B}\right)$ to the measurements of at least another qubit. On the other hand, $\rho^{D}, \rho^{E}, \rho^{F}$ always yield perfectly correlated results. Note that mixed states can lead to correlated results, when they express perfect classical correlations as $\rho^{D}$ does.

Let us formalize the ideas emerging from the former example. Consider a multipartite system composed of $m$ isomorphic subsystems, labeled with indices $i=1, \ldots, m$, with associated Hilbert space $\mathcal{H}^{m}:=\mathcal{H}_{1} \otimes \ldots \otimes \mathcal{H}_{m} \simeq \mathcal{H}^{\otimes m}$, with $\operatorname{dim}\left(\mathcal{H}_{i}\right)=\operatorname{dim}(\mathcal{H})=n$ and $n \geqslant 2$. We shall refer to this multipartite system as to our quantum network. For any operator $X \in \mathfrak{B}(\mathcal{H})$, we will denote by $X^{\otimes m}$ the tensor product $X \otimes$ $X \otimes \ldots \otimes X$ with $m$ factors. Given an operator $\sigma \in \mathfrak{B}(\mathcal{H})$, we denote by $\sigma^{(i)}$ the local operator

$$
\sigma^{(i)}:=I^{\otimes(i-1)} \otimes \sigma \otimes I^{\otimes(m-i)} .
$$

Permutations of quantum subsystems are expressed by a unitary operator $U_{\pi} \in \mathfrak{U}(\mathcal{H})$, which is uniquely defined by

$$
U_{\pi}\left(X_{1} \otimes \ldots \otimes X_{m}\right) U_{\pi}^{\dagger}=X_{\pi(1)} \otimes \ldots \otimes X_{\pi(m)}
$$

for any operators $X_{1}, \ldots X_{m}$ in $\mathfrak{B}(\mathcal{H})$, where $\pi$ is a permutation of the first $m$ integers. A state or observable is said to be permutation invariant if it commutes with all the subsystem permutations. It is worth noting that given any observable $Q \in$ $\mathfrak{H}\left(\mathcal{H}^{m}\right)$, we can define a permutation invariant observable $X$ by considering

$$
X=\frac{1}{m !} \sum_{\pi \in \mathfrak{P}} U_{\pi}^{\dagger} Q U_{\pi} .
$$

Definition $1(\sigma E C)$ : Given $\sigma \in \mathfrak{B}(\mathcal{H})$, a state $\rho \in \mathfrak{D}\left(\mathcal{H}^{m}\right)$ is in $\sigma$-Expectation Consensus ( $\sigma \mathrm{EC})$ if

$$
\operatorname{Tr}\left(\sigma^{(1)} \rho\right)=\ldots=\operatorname{Tr}\left(\sigma^{(k)} \rho\right) .
$$

The reduced state (analog of a marginal distribution) of subsystem $k$ for an overall system state $\rho$ is defined by $\bar{\rho}_{k}=$ $\operatorname{Tr}_{\left(\otimes_{j \neq k} \mathcal{H}_{j}\right)}(\rho)$.

Definition 2(RSC): A state $\rho \in \mathfrak{D}\left(\mathcal{H}^{m}\right)$ is in Reduced State Consensus (RSC) if

$$
\bar{\rho}_{1}=\bar{\rho}_{2}=\ldots=\bar{\rho}_{m} .
$$

Definition 3 (SSC): A state $\rho \in \mathfrak{D}\left(\mathcal{H}^{m}\right)$ is in Symmetric State Consensus (SSC) if, for each unitary permutation $U_{\pi}$

$$
U_{\pi} \rho U_{\pi}^{\dagger}=\rho .
$$

Definition 4: $(\sigma \mathrm{SMC})$ Given an observable $\sigma$ with spectral decomposition $\sigma=\sum_{j=1}^{d} s_{j} \Pi_{j} \in \mathfrak{H}(\mathcal{H}),{ }^{2}$ a state $\rho \in \mathfrak{D}\left(\mathcal{H}^{m}\right)$

\footnotetext{
${ }^{1}$ The set $\left\{c_{1}, c_{2}, c_{3}, \ldots\right\}^{\times n}$ is the Cartesian product of $\left\{c_{1}, c_{2}, c_{3}, \ldots\right\}$ by itself $n$ times, i.e., the set of $n$-tuples with components taken from $\left\{c_{1}, c_{2}, c_{3}, \ldots\right\}$.

${ }^{2}$ We here assume that all $s_{j}$ are different, so $d \leq n$. See Appendix A2 for more on observables and related stochastic measurement results.
} 
is in Single $\sigma$-Measurement Consensus ( $\sigma \mathrm{SMC})$ if

$$
\operatorname{Tr}\left(\Pi_{j}^{(k)} \Pi_{j}^{(\ell)} \rho\right)=\operatorname{Tr}\left(\Pi_{j}^{(\ell)} \rho\right)
$$

for all $k, \ell \in\{1, \ldots, m\}$, and for each $j$.

The definition of $\sigma \mathrm{SMC}$ requires that the outcomes of $\sigma$ measurements on different subsystems be exactly the same for each trial. Indeed, in this last definition, the right-hand side of (4) is the probability of obtaining $s_{j}$ as a measurement result on both subsystems $\ell$ and $k$ (note that $\Pi_{j}^{(k)}$ and $\Pi_{j}^{(\ell)}$ commute, so this joint measurement $\Pi_{j}^{(k)} \Pi_{j}^{(\ell)}$ is well-defined). Then if (4) holds, the probability of $s_{j}$ on $k$ conditional to observing $s_{j}$ on $\ell$ is one (assuming that $\Pi_{j}^{(\ell)} \rho \Pi_{j}^{(\ell)} \neq 0$; that special case is trivial and can be treated separately).

All the states in our example satisfy $\sigma_{z} \mathrm{EC}$, all but $\rho^{A}$ satisfy RSC, $\rho^{C}$ to $\rho^{F}$ satisfy SSC, and $\rho^{D}$ to $\rho^{F}$ satisfy $\sigma_{z}$ SMC. There obviously seems to be a hierarchy in these definitions, and the following properties are meant to better characterize them.

Theorem 1: The following chain of implications holds:

$$
\mathrm{SSC} \Longrightarrow \mathrm{RSC} \Longrightarrow \sigma \mathrm{EC}
$$

while the converse implications are not true in general.

Proof-SSC $\Longrightarrow R S C$ : If $U_{\pi} \rho U_{\pi}^{\dagger}=\rho$ for each permutation, consider in particular $U_{(\ell, k)}$ that swaps subsystems $\ell$ and $k$. Then

$$
\bar{\rho}_{k}=\operatorname{Tr}_{\otimes_{j \neq k} \mathcal{H}_{j}}(\rho)=\operatorname{Tr}_{\otimes_{j \neq k} \mathcal{H}_{j}}\left(U_{(\ell, k)} \rho U_{(\ell, k)}^{\dagger}\right)=\bar{\rho}_{\ell}
$$

and the reasoning can be repeated for any pair. $R S C \Longrightarrow \sigma E C$ is immediate by definition. States $\rho^{B}$ and $\rho^{A}$ from Example 1 provide counterexamples for the converse of the first and of the second implication, respectively.

In order to obtain converse relations one has to add some hypotheses:

Proposition 1: The following hold:

1) A state is RSC if and only if it is $\sigma \mathrm{EC}$ for all $\sigma \in \mathfrak{H}(\mathcal{H})$;

2) If $\rho$ is in RSC, with $\bar{\rho}_{k}$ a pure state for each $k$, then it is also in SSC.

The proof is given in Appendix B. We next characterize the notion of $\sigma \mathrm{SMC}$, and explore its relationship with the other notions. Consider the set of projections $\left\{\Pi_{j}\right\}_{j=1}^{d}$ as in Definition 4, and let us define $\Pi_{\text {sym }}=\sum_{j=1}^{d} \Pi_{j}^{\otimes m}$.

Theorem 2: A state is in $\sigma \mathrm{SMC}$ if and only if it holds

$$
\operatorname{Tr}\left(\Pi_{\text {sym }} \rho\right)=1
$$

or equivalently

$$
\Pi_{\mathrm{sym}} \rho \Pi_{\mathrm{sym}}=\Pi_{\mathrm{sym}} \rho=\rho .
$$

Furthermore:

a) $\sigma \mathrm{SMC}$ implies $\sigma \mathrm{EC}$;

b) $\sigma \mathrm{SMC}$ for $\sigma$ with non-degenerate spectrum implies RSC;

c) $\sigma \mathrm{SMC}$ for $\sigma$ with non-degenerate spectrum implies SSC;

d) The converse implications of a), b), or c) do not hold;

e) It is impossible for a state to be $\sigma \mathrm{SMC}$ with respect to all $\sigma \in \mathfrak{H}(\mathcal{H})$.

The proof is given in Appendix B. We thus have, as could be expected, that $\sigma \mathrm{SMC}$ is in general a stronger notion of consensus, as long as $\sigma$ has non-degenerate spectrum.
Remark: It is worth remarking how all these definitions could be given for classical systems, in the context of consensus for random variables or for probability distributions of the state values. In this case, for example, $\sigma \mathrm{EC}$ would require the expectation of a set of random variables, each one associated to a subsystem, to be the same in all subsystems; RSC would require the marginal distributions on each subsystem to be equal; and SSC would require that the joint probability distribution is invariant with respect to subsystem permutations.

\section{QUANTUM EVOLUTIONS ON NETWORKS AND Their Asymptotic Properties}

So far we have been concerned with discussing "static" properties of consensus states. However, the core of the problem is the design of discrete-time dynamical systems (or algorithms) that drive the system to consensus. In the following sections, we will establish which dynamics are needed to drive an arbitrary initial state towards a consensus state. In addition to this, as in the classical consensus problems, we shall require the final state to preserve or express some property dependent on the initial state. For example, when classical average consensus is reached, each agent locally holds ("has computed") the average of the initial state values, which is a global property. In the quantum case as well, a goal when reaching consensus would often be to retrieve, in the final state of any local subsystem, some global information about the initial state.

\section{A. Classical Dynamics and Locality}

For classical consensus, the starting point is a first-order integrator dynamics for each individual agent, of the type

$$
x_{k}(t+1)=x_{k}(t)+u_{k}(t) \quad \text { or } \quad \frac{d}{d t} x_{k}(t)=u_{k}(t)
$$

for $k=1,2, \ldots, m$. In this paper we focus on the discretetime case. The inputs $u_{k}(t)$ for the agents can take different forms, but they are all based on local information. A notion of locality is traditionally introduced by specifying a directed graph $G(V, E)$ whose vertices are the agents $1,2, \ldots, m$ and where an edge $(j, k) \in E$ (ordered pair of vertices) indicates that agent $j$ can send information to agent $k$. Then for each $k$, input $u_{k}$ is restricted to depend only on $x_{k}$ and on the states $x_{j}$ of agents $j$ for which $(j, k) \in E$. We consider $u_{k}$ written as the sum of contributions from different edges, $u_{k}(t)=$ $\sum_{j:(j, k) \in E} f_{j, k}\left(x_{k}(t), x_{j}(t)\right)$. Weights $w_{(j, k)}$ can be associated to the edges to model their relative strengths. Besides the given asymmetry through edge weights, one usually forbids $u_{k}$ to explicitly use agent identifiers and apply a different treatment to information coming from or going towards different neighbors. This implies

$$
u_{k}(t)=\sum_{j:(j, k) \in E} w_{(j, k)} f\left(x_{j}, x_{k}\right)
$$

where now $f$ must be independent of $j, k$. Finally, one may request that the influence of agent $j$ on agent $k$ be exactly equivalent to the reverse influence, of agent $k$ on agent $j$. Then $u_{k}$ of the type (8) features symmetric weights, $w_{(j, k)}=w_{(k, j)}$ leading to an undirected weighted graph-and the antisymmetry property $f(x, y)=-f(y, x)$ for any $x, y \in \mathbb{R}^{n}$. Such evolution would preserve the average of the state values. 
More generally, locality can be defined with quasi-local operators. Instead of considering a graph, we define a set of neighborhoods $\mathcal{N}_{j} \subseteq\{1, \ldots, m\}$ for $j=1, \ldots, M$, and a quasi-local operator is one that leaves all subsystems unchanged except those of one neighborhood $\mathcal{N}_{j}$. Then a simple dynamics with local coupling would write

$$
x(t+1)=\sum_{j=1}^{M} V_{j}(x(t))
$$

where $V_{j}: \mathbb{R}^{m n} \rightarrow \mathbb{R}^{m n}$ for each $j$ is a quasi-local operator acting on the neighborhood $\mathcal{N}_{j}$. Treating all involved agents equivalently can be formulated as requiring that for each $j$, the quasi-local operator satisfies $P V_{j}=V_{j} P$ for all agent permutations $P$. The classical consensus algorithm $(7),(8)$ is obtained by taking two-agent neighborhoods only, and identifying each $\mathcal{N}_{j}$ with an edge of the graph.

\section{B. Quantum Dynamics and Locality}

According to Schrödinger's equation, isolated quantum systems evolve unitarily [20], [21]. However, unitary dynamics are not enough when we are interested in studying or engineering convergence features for a quantum system. A more general framework that includes (Markovian) open-system evolutions is offered by quantum channels [21], [22], that is, linear, completely positive (CP) and trace preserving (TP) maps from density operators to density operators $\mathcal{E}: \mathfrak{D}\left(\mathcal{H}^{m}\right) \rightarrow \mathfrak{D}\left(\mathcal{H}^{m}\right)$. It can be shown that such maps admit an operator sum representation (OSR), also known as Kraus decomposition:

$$
\mathcal{E}(\rho)=\sum_{k=1}^{K} A_{k} \rho A_{k}^{\dagger} \quad \text { with } \quad \sum_{k=1}^{K} A_{k}^{\dagger} A_{k}=I
$$

where $K \leqslant(\operatorname{dim}(\mathcal{H}))^{2}$. The representation is not unique, however the relation between all the possible different representations is well known (see [21, Theorem 8.2]). A CPTP map is said unital if $\mathcal{E}(I)=I$. These maps represent the quantum equivalent of doubly-stochastic transition matrices for Markov processes. A particular set of unital quantum channels is given by random unitaries [23]. A channel belongs to this class when it admits an OSR with $K$ operators $A_{k}=\sqrt{p_{k}} U_{k}$, with $U_{k} \in$ $\mathfrak{U}\left(\mathcal{H}^{m}\right)$ and $p_{k} \geq 0$ such that $\sum_{k=1}^{K} p_{k}=1$ :

$$
\mathcal{E}(\rho)=\sum_{k=1}^{K} p_{k} U_{k} \rho U_{k}^{\dagger}
$$

Such a map can be thought of as a probabilistic mixture of unitary evolutions.

Given a CPTP map $\mathcal{E}$, we can define its dual map with respect to the Hilbert-Schmidt inner product $\mathcal{E}^{\dagger}: \mathfrak{B}(\mathcal{H}) \rightarrow$ $\mathfrak{B}(\mathcal{H})$ through the relation:

$$
\operatorname{Tr}[A \mathcal{E}(\rho)]=\operatorname{Tr}\left[\mathcal{E}^{\dagger}(A) \rho\right] .
$$

This dual map is still linear and completely positive, while the fact that $\mathcal{E}$ is trace preserving implies that $\mathcal{E}^{\dagger}$ is always unital. Considering the dynamics in the dual picture, i.e., with timeinvariant states and maps acting on the observables, is called Heisenberg's picture in the physics literature and provides an equivalent description of quantum system evolution.
We now introduce locality notions for the quantum network. Consider the multipartite system introduced in Section II-B: following [11], we say that an operator in $\mathfrak{B}(\mathcal{H})$ is quasilocal if it acts nontrivially only on one neighborhood $\mathcal{N}_{j} \subseteq$ $\{1, \ldots, m\}$ :

Definition 5 (Quantum Quasi-Local Operator): An operator $V$ is quasi-local with respect to a set of neighborhoods $\left\{\mathcal{N}_{j}, j=\right.$ $1,2, \ldots, M\}$, if and only if there exists $j \in\{1,2, \ldots, M\}$ such that

$$
V=V_{\mathcal{N}_{j}} \otimes I_{\overline{\mathcal{N}}_{j}}
$$

where, with a slight abuse of notation, $V_{\mathcal{N}_{j}}$ accounts for the nontrivial action on $\mathcal{H}_{\mathcal{N}_{j}}$ and $I_{\overline{\mathcal{N}_{j}}}=\bigotimes_{k \notin \mathcal{N}_{j}} I_{k}$.

\section{Timing of Operations and Evolution Types}

In classical consensus, an important aspect is that the graph (and the related interaction law) can be time-varying. For instance one can assume that all edges are activated for the whole time (synchronous update), at the other extreme that they are activated one at a time, or some at each time (asynchronous update), according to some predefined time-varying sequence or by random selection of edges. Interestingly, convergence properties for all these cases can be linked to the connectedness of the "average graph" [24].

In the quantum case also this distinction can be made. The elementary dynamical interaction that we consider, replacing "one edge" of the classical case, is a CPTP map involving one neighborhood only:

$$
\mathcal{E}_{\mathcal{N}_{j}}(\rho)=\sum_{k=1}^{K} p_{k} V_{k}(t) \rho V_{k}^{\dagger}(t)
$$

where all the $V_{k}(t) \in \mathfrak{U}\left(\mathcal{H}^{m}\right)$ are quasi-local with respect to the neighborhood $\mathcal{N}_{j}, j \in\{1,2, \ldots, M\}$. One of the reasons for focusing on this class of evolutions stems directly from applications: methods for implementing unitary evolutions, as well as related unital channels with the aid of some ancillary systems, are available in a number of diverse experimental settings. On the other hand, constructing arbitrary quantum channels is a more challenging task [25], and can be generally done with good approximation only in the limit of fast control and/or short time scales [7]. The building block (13) can lead to different evolutions for the whole system, depending on neighborhood selection:

- Random single interactions: at each time $t$ one neighborhood $\mathcal{N}_{j(t)}$ is selected at random, $j(t)$ being a singlevalued random variable onto the neighborhood index set.

- Cyclic single interactions: at each time $t$ one neighborhood $\mathcal{N}_{j(t)}$ is selected deterministically, for example periodically cycling between the available $j$.

- Random or cyclic asynchronous interactions: similar to the previous options, but a subset of several neighborhoods is selected at each time $t$. We can request the selected neighborhoods to be disjoint or not. This choice may have consequences for the implementation and convergence speed, but not for the convergence property of our algorithm, so we will not consider it further.

- Synchronous interactions: all the available interactions are activated at each time, weighted by some $q_{j} \geq 0$ with 
$\sum_{j=1}^{M} q_{j}=1$ to maintain a trace-preserving map:

$$
\mathcal{E}(\rho)=\sum_{j=1}^{M} q_{j} \mathcal{E}_{\mathcal{N}_{j}}(\rho) .
$$

- Expected evolution: we study the evolution in expectation of the random interaction protocol which selects neighborhood $\mathcal{N}_{j}$ with probability $q_{j}$ at each $t$. Remarkably, the evolution to $\rho_{t+1}$ given $\rho_{t}$ then follows the same law (14) as the synchronous case. Note that convergence of the expected evolution to consensus does not guarantee (at all) that a(ny) single evolution, determined by a realization of the random process $\{j(t)\}_{t>0}$, would converge to consensus. Nevertheless, the first order statistics of any measurements performed at any time on the system will be exactly the same for (14) as for the associated random evolution. In this sense, convergence in expectation is indistinguishable from trajectory-wise convergence.

The last two cases involve a time-independent map. Another time-independent map is obtained if we consider cyclic interactions of period $T$ and we focus on the state at the end of every cycle:

$$
\rho_{t+T}=\mathcal{E}_{C}\left(\rho_{t}\right)=\mathcal{E}_{\mathcal{N}_{T}} \circ \ldots \circ \mathcal{E}_{\mathcal{N}_{1}}\left(\rho_{t}\right) .
$$

The consensus goal can now be specified formally.

Let $d\left(\rho_{a}, \mathcal{C}\right)=\inf _{\rho \in \mathcal{C}}\left\|\rho_{a}-\rho\right\|$, where $\mathcal{C} \subset \mathfrak{D}(\mathcal{H})$ and $\|\cdot\|$ is any $p$-norm on $\mathfrak{B}(\mathcal{H})$. Given a sequence of channels $\left\{\mathcal{E}_{t}(\cdot)\right\}_{t=0}^{\infty}$, define $\hat{\mathcal{E}}_{t}\left(\rho_{0}\right)=\rho_{t}=\mathcal{E}_{t} \circ \mathcal{E}_{t-1} \circ \cdots \circ \mathcal{E}_{1}\left(\rho_{0}\right)$, and $\mathcal{C}_{\sigma \mathrm{EC}}$ to be the set of states in $\sigma \mathrm{EC}$ consensus.

Definition 6 (Asymptotic Consensus): A sequence of channels $\left\{\mathcal{E}_{t}(\cdot)\right\}_{t=0}^{\infty}$, is said to asymptotically achieve $\sigma E C$ if

$$
\lim _{t \rightarrow \infty} d\left(\hat{\mathcal{E}}_{t}\left(\rho_{0}\right), \mathcal{C}_{\sigma \mathrm{EC}}\right)=0
$$

for all initial states $\rho_{0}$.

The same definition holds for RSC, SSC, and $\sigma$ SMC by substituting the corresponding state sets in (16).

Definition 7 (Asymptotic Average Consensus): We say that the sequence of channels $\left\{\mathcal{E}_{t}(\cdot)\right\}_{t=0}^{\infty}$ asymptotically achieves $S$-average $\sigma E C$ for some $S \in \mathfrak{H}\left(\mathcal{H}^{m}\right)$ if it asymptotically achieves $\sigma \mathrm{EC}$ and for all $\rho_{0}$, it holds

$$
\begin{aligned}
\lim _{t \rightarrow \infty} \operatorname{Tr}\left(\sigma \bar{\rho}_{\ell}(t)\right) & =\lim _{t \rightarrow \infty} \operatorname{Tr}\left(\sigma^{(\ell)} \rho(t)\right)=\lim _{t \rightarrow \infty} \operatorname{Tr}(S \rho(t)) \\
& =\operatorname{Tr}\left(S \rho_{0}\right)
\end{aligned}
$$

for all $\ell \in\{1, \ldots, m\}$. The same definition holds for $\sigma$ SMC .

We say that the sequence of channels $\left\{\mathcal{E}_{t}(\cdot)\right\}_{t=0}^{\infty}$ asymptotically achieves $S$-average $R S C$ (resp. SSC) if it asymptotically achieves RSC (resp. SSC) and for $S \in \mathfrak{H}\left(\mathcal{H}^{m}\right)$ there exists a $\sigma \in \mathfrak{H}(\mathcal{H})$ such that (17) holds for all $\rho_{0}$.

By expressing the action of quantum channels in the dual (Heisenberg) picture, it is possible to obtain a clear characterization of the dynamics that satisfy (17).

Proposition 2: Consider a sequence of CPTP channels $\left\{\mathcal{E}_{t}(\cdot)\right\}_{t=0}^{\infty}$, and call $\hat{\mathcal{E}}_{t}=\mathcal{E}_{t} \circ \mathcal{E}_{t-1} \circ \ldots \circ \mathcal{E}_{1}$. The associated dynamics satisfies (17) if and only if

$$
S=\lim _{t \rightarrow \infty} \hat{\mathcal{E}}_{t}^{\dagger}(S) \text { and } \lim _{t \rightarrow \infty} \hat{\mathcal{E}}_{t}^{\dagger}\left(\sigma^{(\ell)}\right)=S
$$

for $\ell=1,2, \ldots, m$, where $\hat{\mathcal{E}}_{t}^{\dagger}=\mathcal{E}_{1}^{\dagger} \circ \mathcal{E}_{2}^{\dagger} \circ \ldots \circ \mathcal{E}_{t}^{\dagger}$.
Proof: The conditions (18) clearly imply (17). On the other hand, if (17) holds for all $\rho_{0}$, it is easy to obtain (18) by duality, taking the limit inside the trace functional.

The first of the equalities in (18) holds in particular for the natural situation where $\mathcal{E}_{t}^{\dagger}(S)=S$ for all $t$. Similarly to the classical case, average quantum consensus algorithms could be a useful tool towards locally estimating collective quantities of an ensemble of many subsystems. Typically in large-ensemble quantum experiments, only few subsystems might be accessible by a measurement apparatus, and then applying a robust consensus procedure to the final state of the system could allow local measurements to provide a kind of "average state" knowledge of the whole ensemble-including potentially quantum correlations that survive throughout the network, e.g., satisfying pairwise Bell-inequalities, if several subsystems can be conditionally measured. Section V discusses this in more detail.

\section{A Gossip Algorithm For QuAntum Consensus}

We now propose actual interactions that drive the quantum network to average consensus. As a building block, we focus on the interaction between two subsystems while the others remain unchanged; all neighborhood-activation options build on this elementary case, as explained above.

\section{A. Another Viewpoint on the Classical Gossip Algorithm}

The standard linear consensus algorithm corresponds to (7), (8) with $f(x, y)=\alpha(x-y)$. Its form with a single interaction activated at any time - also called gossip algorithm -is usually described as follows [26]. At each iteration, a single edge $(j, k)$ is selected from the set $E(t)$ of available edges at that time. The associated agents move towards each other or their mean value, according to

$$
\begin{aligned}
x_{j}(t+1) & =x_{j}(t)+\alpha\left(x_{k}(t)-x_{j}(t)\right) \\
& =(1-\beta) x_{j}(t)+\beta \frac{x_{j}(t)+x_{k}(t)}{2} \\
x_{k}(t+1) & =x_{k}(t)+\alpha\left(x_{j}(t)-x_{k}(t)\right) \\
& =(1-\beta) x_{k}(t)+\beta \frac{x_{j}(t)+x_{k}(t)}{2} \\
x_{\ell}(t+1) & =x_{\ell}(t) \text { for all } \ell \notin\{j, k\}
\end{aligned}
$$

where $\alpha \in(0,1)$ to have meaningful results, ${ }^{3}$ and $\beta=2 \alpha$. An alternative viewpoint on this behavior is that the interacting agents take a weighted average between two discrete operations: [keep your state] and [swap your state]; namely,

$$
\begin{aligned}
\left(x_{j}(t+1), x_{k}(t+1)\right)= & (1-\alpha)\left(x_{j}(t), x_{k}(t)\right) \\
& +\alpha\left(x_{k}(t), x_{j}(t)\right) \\
x_{\ell}(t+1)= & x_{\ell}(t) \text { for all } \ell \notin\{j, k\} .
\end{aligned}
$$

This latter viewpoint turns out to have a natural quantum counterpart. Working with neighborhoods, one could also apply multi-agent permutations, e.g.,

$$
\begin{aligned}
\left(x_{j}, x_{k}, x_{l}\right)_{t+1}=(1-\alpha-\beta)\left(x_{j}, x_{k}, x_{l}\right)_{t} & +\alpha\left(x_{k}, x_{l}, x_{j}\right)_{t} \\
& +\beta\left(x_{l}, x_{j}, x_{k}\right)_{t} .
\end{aligned}
$$

The following result (see, e.g., [26]) characterizes convergence to consensus with the gossip algorithm. While it is a known

\footnotetext{
${ }^{3}$ That is, the new states are an interpolation and not extrapolation between $x_{j}(t)$ and $x_{k}(t)$.
} 
result, we nonetheless provide a proof that will be useful to our aim, i.e., proving the convergence of the quantum gossip algorithm, and makes our presentation more self-contained.

Proposition 3: Consider $G(V, E)$ an undirected graph that is connected, i.e., for any pair of vertices $a, b \in V$, there exists a sequence of vertices $v_{0}=a, v_{1}, v_{2}, \ldots, v_{n-1}, v_{n}=b$ such that $\left(v_{k-1}, v_{k}\right) \in E$ for all $k=0,1, \ldots, n$. If one step of the classical gossip algorithm (19) is applied at each time, selecting the updated edge by cyclically running through all the edges of $G(V, E)$, then the system exponentially converges to average consensus. Moreover, if the updated edge $(j, k)$ is selected randomly according to a fixed probability distribution $\left\{q_{j, k}\right\}$, with all $q_{j, k}>0$, then asymptotic average consensus is ensured with probability one, in the sense that: for any $\delta, \varepsilon>0$, there exists a time $T>0$ such that

$$
\left.\mathbb{P}\left[\| x_{k}(T)-\bar{x}\right)\left\|^{2}>\varepsilon\right\| x_{k}(0)-\bar{x} \|^{2}\right]<\delta
$$

where $\mathbb{P}$ denotes the probability measure induced by the randomization, $\|x\|^{2}=\sum_{k=1}^{m} x_{k}^{T} x_{k}$ and $\bar{x}=(1 / m) \sum_{k=1}^{m} x_{k}(t)=$ $\sum_{k=1}^{m} x_{k}(0)$ for any choice of the edges.

Proof: We denote $x^{T} x=\|x\|^{2}$ for short and $|E|$ the number of edges in $G(V, E)$. At any step of the gossip algorithm, $W:=(1 / 2 m) \sum_{k, j=1}^{m}\left\|x_{k}-x_{j}\right\|^{2}=\sum_{k=1}^{m}\left\|x_{k}-\bar{x}\right\|^{2}$ can only remain unchanged (if the two nodes of the selected edge have the same value) or decrease (as soon as an edge with different node values is selected). Therefore, $W$ is a (non-strict) Lyapunov function for the system dynamics, and when the edges of a connected graph are selected in a cyclic way, a direct application of the LaSalle invariance theorem (see, e.g., [27]) shows that the system asymptotically converges to the consensus set. Since the map associated to one full cycle of edge selections is linear and time-invariant, this convergence is exponential. For such convergence to be possible, there must exist some $\lambda>0$ and integer $M>0$ such that $W(T) \leq W(0) \lambda$ if the edge choice between $t=0$ and $t=T=M|E|$ corresponds to $M$ cycles of gossip. When edges are selected randomly, any particular sequence of $b$ consecutive edge selections has a probability greater than $\bar{q}^{b}>0$ to appear at least once during any time interval of length at least $b$, where $\bar{q}=\min _{(j, k) \in E} q_{j, k}$. In particular, if we target $W(T)<\varepsilon W(0)=\lambda^{r} W(0)$, we can say that there is a probability at least $\bar{q}^{r M|E|}$ to select $r$ times a succession of $M$ cyclic interactions between $t=t_{0}$ and $t=$ $t_{0}+r M|E|$. If this happens once, any preceding or following edge choice can only improve $W$ (because of our first statement in this proof). We conclude by noting that over a time interval $\operatorname{br} M|E|$, there is then a probability $<\left(1-\bar{q}^{r M|E|}\right)^{b}$ to have never selected $r$ times a succession of $M$ cyclic interactions, and thus potentially miss $W(T)<\varepsilon W(0)$; the probability that this happens can be made arbitrarily small by taking $b$ (thus $T$ ) sufficiently large.

\section{B. Quantum Gossip Interactions}

Let us introduce a way to implement gossip-type interactions in a fully quantum way. In a controlled quantum network, one can typically engineer unitary transformations that implement the "identity" evolution and the swapping of two neighboring subsystem states; let us denote the latter operator by $U_{(j, k)}$ for swapping subsystems $j$ and $k$. To develop our analysis, it will be convenient to introduce the graph $G$ associated to the multipartite system: its nodes $1, \ldots, m$ correspond to the "physical" subsystems, the edge $(j, k)$ is included if the subsystems $j$ and $k$ have a nonzero probability to interact.

Assume edge $(j, k)$ is selected at a certain step $t$. We then consider an auxiliary two-level system $\mathcal{Q}$ and the joint unitary evolution $I \otimes\left|\xi_{I}\right\rangle\left\langle\xi_{I}\left|+U_{(j, k)} \otimes\right| \xi_{S}\right\rangle\left\langle\xi_{S}\right|$ of the quantum network and the auxiliary system. This conditionally associates the two operations $I, U_{(j, k)}$ on the network to the orthogonal states $\left|\xi_{I}\right\rangle$ and $\left|\xi_{S}\right\rangle$ of $\mathcal{Q}$. Denoting by $\rho$ the initial state of the quantum network and by $\rho_{\xi}=(1-\alpha)\left|\xi_{I}\right\rangle\left\langle\xi_{I}|+\alpha| \xi_{S}\right\rangle\left\langle\xi_{S}\right|+$ $\beta\left|\xi_{S}\right\rangle\left\langle\xi_{I}\left|+\beta^{*}\right| \xi_{I}\right\rangle\left\langle\xi_{S}\right|$ the generic initial state of $\mathcal{Q}$, the joint state after the evolution gets

$$
\begin{aligned}
\rho \otimes \rho_{\xi} \rightarrow(1-\alpha) \rho \otimes\left|\xi_{I}\right\rangle\left\langle\xi_{I}\left|+\alpha U_{(j, k)} \rho U_{(j, k)}^{\dagger} \otimes\right| \xi_{S}\right\rangle\left\langle\xi_{S}\right| \\
+\beta U_{(j, k)} \rho \otimes\left|\xi_{S}\right\rangle\left\langle\xi_{I}\left|+\beta^{*} \rho U_{(j, k)}^{\dagger} \otimes\right| \xi_{I}\right\rangle\left\langle\xi_{S}\right| .
\end{aligned}
$$

Taking the partial trace over the auxiliary system, we obtain as evolution for the quantum network a quantum channel that represents our fundamental quantum gossip interaction:

$$
\rho(t+1)=\mathcal{E}_{j, k}(\rho(t))=(1-\alpha) \rho(t)+\alpha U_{(j, k)} \rho(t) U_{(j, k)}^{\dagger}
$$

with $\alpha \in(0,1)$. Note that the conditional swapping only involves purely local interactions among subsystems $j$ and $k$ of the quantum network, plus the auxiliary system $\mathcal{Q}$ associated to this pair. The state of $\mathcal{Q}$ after interaction is discarded; hence, it does not need measurement equipment. Moreover, any choice of $\alpha \notin\{0,1\}$ is sufficient to introduce some degree of dissipation (non-unitary evolution) on $\rho(t)$, which is necessary for convergence [9]. In accurately controlled settings [8], one may assume to have an actual resettable ancillary system associated to each link, or one or more "moving" ancillary systems that activate the desired links. Then resetting the ancilla to an initial state with $\alpha=1 / 2$ would optimize quasi-local mixing.

\section{Convergence to Consensus}

We study convergence under three types of gossip dynamics: cyclic interactions, expectation of random interactions, and trajectory-wise for the random interactions. In all these cases, quantum gossip can be described by unital CPTP maps. We begin by recalling a characterization of the fixed points of such maps (see, e.g., [4]).

Proposition 4: Let $\left\{V_{i}\right\}_{i=1}^{K}$ the Kraus decomposition of a unital $\mathrm{CP}$ map $\mathcal{E}(\cdot)$ and define

$\mathcal{A}_{\mathcal{E}}=\left\{X \in \mathfrak{B}\left(\mathcal{H}^{m}\right) \mid X V_{i}-V_{i} X=0 \forall i=1, \ldots, K\right\}$.

Then $\bar{X} \in \mathfrak{B}\left(\mathcal{H}^{m}\right)$ is a fixed point of $\mathcal{E}$, i.e., $\mathcal{E}(\bar{X})=\bar{X}$, if and only if $\bar{X} \in \mathcal{A}_{\mathcal{E}}$.

This helps determine the set of fixed points for the CP maps of interest in quantum gossip.

Lemma 1: Let $U_{(j, k)}$ denote the pairwise swap operation of subsystems $(j, k)$ on $\mathcal{H}^{m}$. If the graph $G$ associated to the system is connected, then the set of fixed points of any CP unital map of the form

$$
\begin{aligned}
\mathcal{E}(X)=q_{0} X+ & \sum_{(j, k) \in E} q_{j, k} U_{(j, k)}^{\dagger} X U_{(j, k)} \\
& \text { with } q_{0}+\sum_{(j, k) \in E} q_{j, k}=1, q_{0},\left\{q_{j, k}\right\}>0
\end{aligned}
$$

coincides with the set of permutation-invariant operators. 


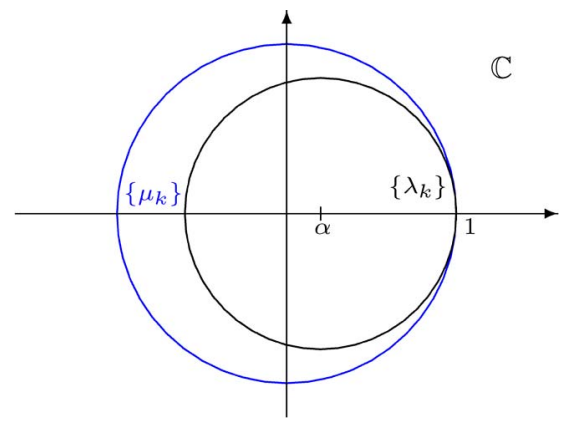

Fig. 1. Delimitation of the (closed) domains for the eigenvalues $\left\{\mu_{k}\right\}$ of $\mathcal{F}$ (blue) and $\left\{\lambda_{k}\right\}$ of $\mathcal{E}$ (red).

Proof: According to Proposition 4 above, the fixed points are the $X$ satisfying $X U_{(j, k)}=U_{(j, k)} X$, or equivalently $U_{(j, k)}^{\dagger} X U_{(j, k)}=X$. The latter expresses that $X$ is invariant with respect to pairwise swaps on all the graph edges. It is well known that sequences of pairwise swaps on the edges of a connected graph generate the full set of permutations on the set of nodes, and so we get the conclusion.

The following lemma shows how the contribution of the identity, i.e., the trivial permutation, in the CP map plays a crucial role in the proof of convergence.

Lemma 2: Consider a linear completely positive map $\mathcal{E}$ on $\mathfrak{B}(\mathcal{H})$ that admits an operator-sum representation $\left\{A_{k}\right\}$ with one operator proportional to identity, i.e., $A_{1}=\sqrt{\alpha} I>0$. Then, if $\lambda$ is an eigenvalue of $\mathcal{E},|\lambda|=1$ implies $\lambda=1$.

Proof: If $\mathcal{E}$ is a CPTP map it is a contraction in trace norm [21], [28], so its eigenvalues $\lambda_{k}$ belong to the closed unit disk. By virtue of the Kraus-Stinespring representation theorem (see, e.g., [22]), also $\mathcal{F}=(1 /(1-\alpha))(\mathcal{E}-\alpha I)$ is CPTP and thus has eigenvalues $\mu_{k}$ in the closed unit disk. Therefore, the eigenvalues $\lambda_{k}=(1-\alpha) \mu_{k}+\alpha$ of $\mathcal{E}=(1-\alpha) \mathcal{F}+\alpha I$ in fact belong to the circle of radius $(1-\alpha)$ centered at $\alpha$, which is strictly inside the unit circle except for a tangency point at $1 \in \mathbb{C}$, see Fig. 1 .

In other words, Lemma 2 excludes eigenvalues of unit norm different from +1 , those which would cause limit cycles.

By combining the above properties, we get the following convergence result for quantum gossip. It shows that $S$ average SSC can be attained for global operators that are the permutation-invariant average of local ones; this is similar to classical gossip, where distributed computation of the average of individual states actually gives access to the value of any linear permutation-invariant function of these states.

Theorem 3: If the graph associated to possible interactions is connected, then the quantum gossip algorithm (21) ensures global convergence towards SSC: deterministically, when the edges on which a gossip interaction occurs at a given time are selected by periodically cycling, in any predefined way, through the set of edges; in expectation, when the edges on which a gossip interaction occurs at a given time are selected randomly from a fixed probability distribution $\left\{q_{j, k}>0 \mid \sum_{(j, k) \in E} q_{j, k}=\right.$ $1\}$; with probability one on any trajectory, with the same edgeselection strategy of the previous point. Explicitly, there exists a state $\rho_{*} \in \mathcal{C}_{S S C}$ for which for any $\delta, \varepsilon>0$, there exists a time $T>0$ such that

$$
\mathbb{P}\left[\operatorname{Tr}\left(\left(\rho(T)-\rho_{*}\right)^{2}\right)>\varepsilon\right]<\delta .
$$

In any of the above cases, the system converges to

$$
\rho_{*}=\frac{1}{m !} \sum_{\pi \in \mathfrak{P}} U_{\pi} \rho_{0} U_{\pi}^{\dagger},
$$

where $\rho_{0}$ is the initial state of the network. Furthermore, $S$ average SSC is attained if and only if $S \in \mathfrak{H}\left(\mathcal{H}^{\otimes m}\right)$ can be written, for some $\sigma \in \mathfrak{H}(\mathcal{H})$, in the form

$$
S=\frac{1}{m} \sum_{i=1}^{m} \sigma^{(i)} \text {. }
$$

Proof: First notice that all the operators in the OSR of the map (15) are self-adjoint. This implies that permutationinvariant observables $S$ are fixed points for the associated dual map, and hence for the gossip interaction associated to any edge $(j, k)$ and $\forall \rho$ :

$$
U_{(j, k)} S U_{(j, k)}^{\dagger}=S \Rightarrow \operatorname{Tr}\left[\mathcal{E}_{j, k}(\rho) S\right]=\operatorname{Tr}\left[\rho \mathcal{E}_{j, k}^{\dagger}(S)\right]=\operatorname{Tr}[\rho S] .
$$

For the cyclic evolution map $\mathcal{E}_{C}$, we notice that all the simple two-subsystem swaps are still present with a weight different from zero in the OSR of the cyclic map (15), thanks to the presence of the identity in the OSR of each gossip interaction step. Therefore, by Lemma 1 the fixed points are the permutationinvariant operators. Now consider the dynamics associated to $\mathcal{E}_{C}$ as a linear, time-invariant map acting on the subspace of hermitian matrices. From Lemma 2 and the fact that the timeinvariant linear map leaves $\mathfrak{D}\left(\mathcal{H}^{m}\right)$ invariant (excluding unstable Jordan blocks), we have that all the modes of the LTI system are asymptotically stable except those corresponding to the fixed-point set, namely the permutation-invariant set: every initial state converges to a fixed point $\rho_{\infty}$ in this set. Thus the SSC set is globally asymptotically stable, and in fact exponentially stable since the map is linear. Let us now prove that $\rho_{\infty}$ has the form (24). For all permutation invariant $X$, from (26) we have that

$$
\operatorname{Tr}\left[X \mathcal{E}_{C}\left(\rho_{0}\right)\right]=\operatorname{Tr}\left[X \rho_{0}\right] \quad \forall t .
$$

Combining the latter with the fact that $\rho_{\infty}$ is permutationinvariant, that the set of all permutations is self-adjoint, and using (3), we get for arbitrary $Q \in \mathfrak{H}\left(\mathcal{H}^{m}\right)$ :

$$
\begin{aligned}
\operatorname{Tr}\left[Q \rho_{\infty}\right] & =\operatorname{Tr}\left[Q \frac{1}{m !} \sum_{\pi \in \mathfrak{P}} U_{\pi} \rho_{\infty} U_{\pi}^{\dagger}\right]=\operatorname{Tr}\left[\frac{1}{m !} \sum_{\pi \in \mathfrak{P}} U_{\pi} Q U_{\pi}^{\dagger} \rho_{\infty}\right] \\
& =\operatorname{Tr}\left[\frac{1}{m !} \sum_{\pi \in \mathfrak{P}} U_{\pi} Q U_{\pi}^{\dagger} \rho_{0}\right]=\operatorname{Tr}\left[\frac{1}{m !} \sum_{\pi \in \mathfrak{P}} Q U_{\pi} \rho_{0} U_{\pi}^{\dagger}\right] .
\end{aligned}
$$

This implies that indeed $\rho_{\infty}=\rho_{*}$ as stated.

For the expectation of random evolution, the CPTP map $\mathcal{E}$ is exactly of the form of Lemma 1 and the same reasoning can be repeated.

For the random trajectory evolution, we repeat a proof similar to that of Proposition 3. Since $\mathcal{E}$ for a single evolution step is linear, self-adjoint with respect to the Hilbert-Schmidt inner product, and thus with eigenvalues in the closed unit disk, it is a contraction for the Frobenius norm distance $\operatorname{Tr}\left(\left(\rho_{A}-\right.\right.$ $\left.\rho_{B}\right)^{2}$ ) between any two states $\rho_{A}, \rho_{B} \in \mathfrak{D}\left(\mathcal{H}^{m}\right)$. Indeed, $\mathcal{E}$ has non-increasing orthonormal modes, so by writing any operator $X \in \mathfrak{H}\left(\mathcal{H}^{m}\right)$ in the modal basis we directly get $\operatorname{Tr}\left(\mathcal{E}(X)^{\dagger} \mathcal{E}(X)\right) \leq \operatorname{Tr}\left(X^{\dagger} X\right)$; taking $X=\rho_{A}-\rho_{B}$ yields the 
contraction. ${ }^{4}$ Now taking in particular $\rho_{A}=\rho$ and $\rho_{B}=\rho_{*}$, we get that the Frobenius distance from $\rho$ to $\rho_{*}$ can never increase. Moreover, by transitivity of the permutation operators, $(1 / m !) \sum_{\pi \in \mathfrak{P}} U_{\pi} \rho U_{\pi}^{\dagger}=(1 / m !) \sum_{\pi \in \mathfrak{P}} U_{\pi} \rho_{0} U_{\pi}^{\dagger}=\rho_{*}$ for any $\rho$ along the trajectory of the gossip algorithm. Now given the convergence under cyclic evolution, there must exist some $\lambda<1$ and integer $M>0$ such that

$$
\operatorname{Tr}\left(\left(\mathcal{E}_{C}^{M}(\rho)-\rho_{*}\right)^{2}\right) \leq \lambda \operatorname{Tr}\left(\left(\rho-\rho_{*}\right)^{2}\right)
$$

for any $\rho$ for which $(1 / m !) \sum_{\pi \in \mathfrak{P}} U_{\pi} \rho U_{\pi}^{\dagger}=\rho_{*}$. The proof then concludes along the same lines as Proposition 3, namely the probability to obtain an edge sequence which includes successions of $M$ cyclic evolutions a sufficiently large number of times to have $\varepsilon$-convergence, gets arbitrarily close to 1 if we wait long enough.

Finally let us prove that we attain $S$-average consensus if and only if $S$ can be decomposed as in (25). We know from the first part of the proof that all permutation-invariant observables $S$ are fixed points for the associated dual map $\mathcal{E}_{t}^{\dagger}$. Then according to Proposition 2 we have $S$-average consensus if and only if there exists a local observable $\sigma$ such that

$$
\lim _{t \rightarrow \infty} \hat{\mathcal{E}}_{t}^{\dagger}\left(\sigma^{(\ell)}\right)=S
$$

for $\ell=1,2, \ldots, m$. Because of (26) and (24), by duality we have that for every local operator $\sigma^{(\ell)}$

$$
\lim _{t \rightarrow \infty} \hat{\mathcal{E}}_{t}^{\dagger}\left(\sigma^{(\ell)}\right)=\frac{1}{m !} \sum_{\pi \in \mathfrak{P}} U_{\pi}^{\dagger} \sigma^{(\ell)} U_{\pi}=\frac{1}{m} \sum_{i=1}^{m} \sigma^{(i)} .
$$

This is the form (25), concluding the proof.

Remark: This shows that the mean value of a (global) observable $S=(1 / m) \sum_{\ell=1}^{m} \sigma^{(\ell)}$, with arbitrary $\sigma$, can be asymptotically retrieved from the state of any single subsystem after having applied one of the quantum gossip algorithms.

On the other hand, unlike for classical consensus, there are permutation-invariant operators that do not attain $S$-average consensus, because they cannot be written in the form (25). This is the case among others if $S$ is orthogonal to the linear span of all the local observables. For instance if $\tilde{S}=\sigma_{z}^{\otimes^{m}}$, given the orthogonal basis $\left\{\sigma_{k}\right\}_{k=0, x, y, z}$ for $\mathfrak{B}(\mathcal{H})$, we have

$\operatorname{Tr}\left[\tilde{S} \sigma_{k}^{(\ell)}\right]=0 \quad \forall k \in\{0, x, y, z\} \quad$ and $\quad \forall l \in\{1, \ldots, m\}$.

Therefore, $\tilde{S}$ cannot be written in the form (25); hence, although $\tilde{S}$ is conserved by the gossip algorithm, the latter cannot lead to $\tilde{S}$-average consensus in the sense of Definition 7.

\section{Classical Equivalent to Observable Consensus Dynamics}

We next show how the quantum gossip algorithm (21) in fact implements in a quantum fashion the classical gossip as we restrict to $\sigma$ EC. According to Definition 1, a quantum state $\rho$ belongs to $\mathcal{C}_{\sigma \mathrm{EC}}$ if:

$$
\operatorname{Tr}\left[\sigma^{(1)} \rho\right]=\ldots=\operatorname{Tr}\left[\sigma^{(m)} \rho\right] .
$$

In view of this, it seems reasonable to attempt a convergence study of the algorithm (21) directly in terms of the evolution of

\footnotetext{
${ }^{4}$ This is analogous to the non-increasing Euclidean norm $x^{T} x=\|x\|^{2}$ under a classical consensus iteration with an undirected graph, and the related contraction of $\left\|x_{A}-x_{B}\right\|^{2}$.
}

the expectation values of the $\sigma^{(\ell)}$ operators. This is not possible for arbitrary quantum evolutions, since a quantum state is far from fully specified by a single set of commuting observable expectations, and different states with the same expectation may lead to very different evolutions. However, our quantum gossip algorithm remarkably allows us to write a model for the average dynamics of the $\sigma^{(\ell)}$ in closed form. More precisely, let us define $z_{\ell}(t):=\operatorname{Tr}\left[\mathcal{E}^{t}\left(\rho_{0}\right) \sigma^{(\ell)}\right]=\operatorname{Tr}\left[\rho_{t} \sigma^{(\ell)}\right]$. Note that for one subsystem swap $U_{(j, k)}$, we have

$$
\operatorname{Tr}\left[\sigma^{(\ell)} U_{(j, k)} \rho U_{(j, k)}^{\dagger}\right]= \begin{cases}z_{\ell} & \text { if } \ell \notin\{j, k\} \\ z_{k} & \text { if } \ell=j \\ z_{j} & \text { if } \ell=k .\end{cases}
$$

According to (31) and (21), the random gossip algorithm update yields, with probability $q_{j, k}$, i.e., when the edge $(j, k)$ is selected

$$
\begin{aligned}
\left(z_{j}(t+1), z_{k}(t+1)\right) & =(1-\alpha)\left(z_{j}(t), z_{k}(t)\right)+\alpha\left(z_{k}(t), z_{j}(t)\right) \\
z_{\ell}(t+1) & =z_{\ell}(t) \quad \text { for all } \quad \ell \notin\{j, k\} .
\end{aligned}
$$

This last expression is exactly the classical gossip algorithm (20). Therefore, Proposition 3 readily implies:

Corollary 1: Under all the various edge selection strategies for quantum consensus algorithm (21), the $z_{\ell}(t), \ell=$ $1,2, \ldots, m$ asymptotically converge towards

$$
\lim _{t \rightarrow \infty} z_{\ell}(t)=\frac{1}{m} \sum_{k=1}^{m} z_{k}(0) \quad \text { for all } \quad \ell \in\{1,2, \ldots, m\} .
$$

We remark that this only proves average $\sigma$-Expectation Consensus of the quantum gossip algorithm, while our previous Theorem 3 shows that the algorithm in fact ensures the stronger average Symmetric State Consensus.

\section{E. Gossip Algorithm Example}

In this section, we briefly discuss the evolution induced by random quantum gossip interactions (21) on a four-qubit network whose associated graph is a path. ${ }^{5}$ We observe its convergence toward average $\sigma \mathrm{EC}$, average RSC and average SSC. In particular we consider as a "target" global observable:

$$
S=\frac{1}{4}\left(\sigma_{z}^{(1)}+\sigma_{z}^{(2)}+\sigma_{z}^{(3)}+\sigma_{z}^{(4)}\right) .
$$

Let the initial state be

$$
\rho=|1,0,1,0\rangle\langle 1,0,1,0|
$$

which is pure, and does not satisfy any of the consensus definitions provided in Section II.

By Theorem 3 the state asymptotically converges to

$$
\begin{aligned}
\rho_{\infty}= & \lim _{t \longrightarrow \infty} \rho(t)=\frac{1}{3 !} \sum_{\pi \in \mathfrak{P}} U_{\pi} \rho_{0} U_{\pi}^{\dagger} \\
=\frac{1}{6}(|1,1,0,0\rangle\langle 1,1,0,0|+| 1,0,1,0\rangle\langle 1,0,1,0| & \quad+|1,0,0,1\rangle\langle 1,0,0,1|+| 0,1,1,0\rangle\langle 0,1,1,0| \\
& \quad+|0,1,0,1\rangle\langle 0,1,0,1|+| 0,0,1,1\rangle\langle 0,0,1,1|) .
\end{aligned}
$$

This expression is clearly invariant under all the subsystem permutations, i.e., $\rho_{\infty}$ is in SSC, and therefore also in RSC

\footnotetext{
${ }^{5}$ I.e. the available neighborhoods, labeling the subsystems as $\{1,2,3,4\}$, are
} $\{1,2\},\{2,3\}$, and $\{3,4\}$. 


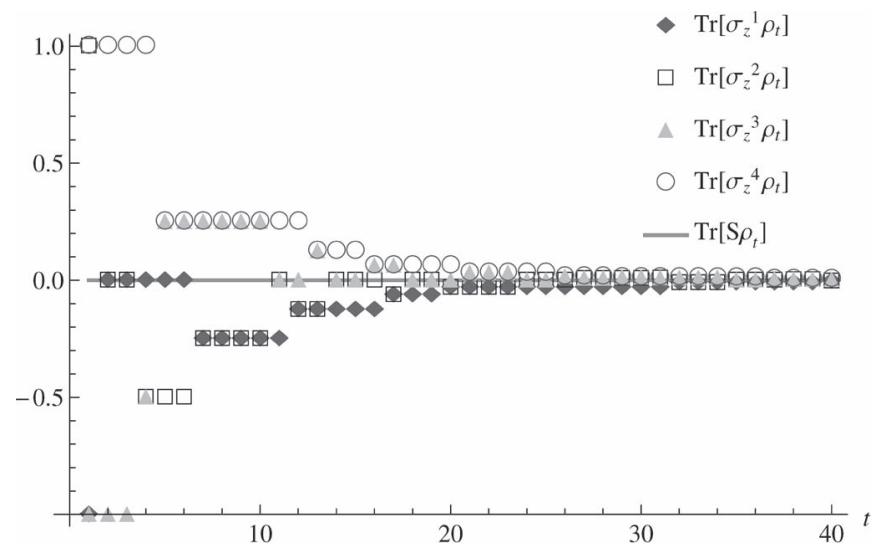

Fig. 2. Evolution toward $\sigma$-Expectation Consensus for a four-qubit network arranged in a path graph.

and $\sigma \mathrm{EC}$ for all $\sigma$. The expectation value of $S$ is preserved at any step, and by Theorem 3 the algorithm drives the system to $S$-average consensus, with $\sigma=\sigma_{z}$.

However, $\rho_{\infty}$ is not in $\sigma \mathrm{SMC}$ for any $\sigma \neq \alpha I$. Indeed, according to Proposition $2, \rho_{\infty}$ is in $\sigma \mathrm{SMC}$ if and only if $\operatorname{Tr}\left[\rho_{\infty} \Pi_{s y m}\right]=1$. Now let $\left\{\Pi_{i}\right\}_{i=1}^{6}$ denote the orthonormal rank-one projectors in (34) and define the orthonormal projector $\bar{\Pi}=\sum_{i=1}^{6} \Pi_{i}$, such that $\rho_{\infty}=(1 / 6) \sum_{i=1}^{6} \Pi_{i}=(1 / 6) \bar{\Pi}$. We then get

$$
\operatorname{Tr}\left[\rho_{\infty} \Pi_{\text {sym }}\right]=\frac{1}{6} \operatorname{Tr}\left[\sum_{i=1}^{6} \Pi_{i} \Pi_{\text {sym }}\right]=\frac{1}{6} \operatorname{Tr}\left[\Pi_{\text {sym }} \bar{\Pi}\right] .
$$

This last expression is equal to 1 if and only if $\operatorname{Tr}\left[\Pi_{\text {sym }} \bar{\Pi}\right]=6$. However, excluding the trivial case $\sigma=\alpha I$, for qubit networks $\Pi_{\text {sym }}$ is always a two dimensional projector, so $\operatorname{Tr}\left[\Pi_{\text {sym }} \bar{\Pi}\right] \leq$ 2 . Hence, $\rho_{\infty}$ cannot be in $\sigma$ SMC for any nontrivial $\sigma$.

Fig. 2 shows the evolution of the expectation values of the local and of the global observables related to $\sigma_{z}$ as the iterations proceed for one run. The edges are selected at random with uniform probability, and the mixing parameter $\alpha$ is taken to be $1 / 2$. With this particular choice, the reduced density operators of two subsystems that have just interacted are equal; this explains why a maximum of three points are visible on the graph at any time. The plot shows that asymptotically the expectation of the local observables $\sigma_{z}$ tend to the expectation value of the global observable $S$, while the expectation value of $S$ is preserved at each step.

\section{Applications}

We now briefly outline three possible applications of the ideas developed in the paper. We want to emphasize the wide flexibility and intrinsic robustness in engineering dynamics that leads to consensus. For example, in our algorithm the strength of mixing, the order of the interactions and the neighborhood topology can be allowed to vary, within the limits imposed by Theorem 3. In this sense, consensus is a robust behavior, that does not have to be tightly controlled. It could, e.g., naturally appear in a large lattice of sites where quantum particles can be found and, because of free or (purposefully) perturbed dynamics, particles are allowed to stochastically move around the lattice; hence, effectively exchanging states between lattice nodes.

In the examples below, we shall assume that such a consensus-yielding process is present in a large network of interest, while accurate control and/or measurement is only possible on a limited number of subsystems - say, those on the boundary of the lattice, or temporarily removed from it to allow interactions with other pieces of laboratory equipments. With experimental quantum systems, this is typically the case when measurement processes are concerned. Thanks to our consensus results, we show how the mixing induced by the consensus dynamics can be exploited to achieve some network-wide tasks with such restricted local control access.

\section{A. Estimation of a Global Variable From a Subsample}

Consider a quantum system composed of a large number $m$ of identical quantum subsystems, initially prepared by some experiment in an unknown global state $\rho$. We are interested in estimating the "average value of a physical property $Q$ over all subsystems," that is the expected value $\bar{q}$ of the observable $\bar{Q}=(1 / m) \sum_{j=1}^{m} Q^{(j)}$, for the state $\rho$, but we are allowed to perform measurements only on a fixed subset of $p \geq 1$ subsystems that are accessible to our measurement apparatus.

Let $q_{j}$ be the random variable (RV) describing the outcome of a local measurement of $Q^{(j)}$ on $\rho$. Since $Q^{(j)}$ and $Q^{(k)}$ commute for $j \neq k$, we can perform a joint measurement of $Q^{(1)}$ to $Q^{(p)}$ on a single realization of $\rho$. A natural estimator for the expectation $\bar{q}=\operatorname{Tr}(\rho \bar{Q})$ would then be of course the sampled average

$$
\hat{q}(p)=\frac{1}{p} \sum_{j=1}^{p} q_{j}
$$

If we can repeat the same experiment, producing $k$ times the same $\rho$ and denoting $q_{j}(z)$ the respective measurement outcomes of $\mathrm{RV} q_{j}$ for $z=1,2, \ldots, k$, then a better estimate would be

$$
\hat{q}(p, k)=\frac{1}{p k} \sum_{j=1}^{p} \sum_{z=1}^{k} q_{j}(z) .
$$

By letting $k$ grow large enough, we can make the variance of $\hat{q}$ arbitrarily small. However, in all practical situations where there might be local variations in the network, the $p$ accessible subsystems are not bound to be representative of the whole ensemble, and $\hat{q}$ is unavoidably biased, unless $p=m$.

This problem is resolved if our gossip-type algorithm can be first enacted on the whole network of $m$ subsystems. Indeed, the measurement statistics obtained from the $p$ fixed subsystems after reaching the consensus state $\rho_{*}=(1 / m !) \sum_{\pi \in \mathfrak{P}} U_{\pi} \rho U_{\pi}^{\dagger}$ are equal to the measurement statistics if we had access to $p$ randomly selected subsystems before consensus. ${ }^{6}$ In particular, the expected value of an estimate $\bar{q}(p)$ of $Q$ from our $p$ subsystems becomes

$$
\begin{aligned}
\mathbb{E}[\bar{q}(p)] & =\frac{1}{p m !} \operatorname{Tr}\left[\sum_{j=1}^{p} \sum_{\pi \in \mathfrak{B}} Q^{(j)} U_{\pi} \rho U_{\pi}^{\dagger}\right]=\frac{1}{p m !} \operatorname{Tr}\left[\sum_{j=1}^{p} \sum_{\pi \in \mathfrak{B}} Q^{(\pi(j))} \rho\right] \\
& =\frac{1}{m} \operatorname{Tr}\left[\sum_{j=1}^{m} Q^{(j)} \rho\right]=\operatorname{Tr}[\bar{Q} \rho]
\end{aligned}
$$

\footnotetext{
${ }^{6}$ This follows from the standard statistical mixture interpretation of a convex combination of density operators.
} 
Thus, $\bar{q}(p)$ provides an unbiased estimator for $\bar{q}$, irrespective of the value of $p$.

Further computations along the lines of statistical sampling without replacement then allow to analyze the variance of $\bar{q}(p)$.

For $k$ independent measurements, the result can be extended as in (37). Note that RSC would be sufficient to guarantee that the estimation is unbiased for any local $Q$. In addition, SSC would allow to generalize the present setting to situations where the local operator $Q$ is replaced by a collective operator on less than $p$ particles. We could then probe different types of average, symmetric correlations in the experimental state $\rho$.

\section{B. Purifying and Cooling of a Sample by Local Feedback Actions}

It is known that unitary control and projective measurements are enough for the preparation of any pure state for a single system [29]. We here show how local access to an arbitrarily small subset of a quantum network, in conjunction with our gossip interactions, is enough to asymptotically prepare a class of factorized, pure states on the whole network.

Consider again a set of $m$ identical subsystems, of which only the first $p$ are accessible via measurements of identical, non-degenerate $Q=\sum_{x=1}^{d} \lambda_{x} \Pi_{x}$. Further assume that, after each measurement, we can apply any desired unitary control on these $p$ subsystems, possibly dependent on the measurement result [25], [29], while some (weaker, unsupervised) control procedure allows us to reach global SSC on the $m$ subsystems. For simplicity we will make explicit reference to our gossip interactions.

We are interested in preparing the whole quantum network in a pure state. It can be shown that any pure, factorized state of the form

$$
\hat{\rho}=|\psi\rangle\langle\psi|\otimes \cdots \otimes| \psi\rangle\langle\psi|
$$

can be asymptotically obtained with the control resources described above, by cyclically iterating the following two steps:

Step I Apply a finite number $M>0$ of gossip interactions.

Step II Perform measurements of $Q$ on each of the $p$ probe subsystems. This brings the network into a state

$$
\Pi_{x_{1}} \otimes \Pi_{x_{2}} \ldots \otimes \Pi_{x_{p}} \otimes \rho_{\backslash p}
$$

where $x_{1}$ to $x_{p}$ are the measurement results and $\rho_{\backslash p}$ is an unknown state on $m-p$ subsystems. Then for each $k=1,2, \ldots, p$, use a unitary control action $U_{k}$ on subsystem $k$ such that $U_{k} \Pi_{x_{k}} U_{k}^{\dagger}=|\psi\rangle\langle\psi|$.

At each iteration of Step II the expectation $V(\rho)=1-$ $\operatorname{Tr}[\hat{\rho} \rho]$ is either left unchanged, if the $p$ subsystems are already all prepared in $|\psi\rangle\langle\psi|$, or else it must decrease. During Step I, $V(\rho)$ is not changed, since $\hat{\rho}$ is a permutation-invariant operator. If the conditions of Theorem 3 hold, then it is easy to show that the largest invariant set for the whole procedure is contained in the kernel of $I-\hat{\rho}$. Hence, by LaSalle invariance theorem we conclude that $\hat{\rho}$ is prepared asymptotically.

If the global Hamiltonian of the network is of the form $H_{\mathrm{tot}}=\sum_{j=1}^{m} Q^{(j)}$ or, more generally, admits a ground state of the form $\hat{\rho}$, then this procedure can be used to obtain groundstate cooling.

By variations of the above protocol, the same control capabilities can be used to engineer dynamics that asymptotically drive the state of the quantum network to have support on an arbitrary target subspace of the network's joint Hilbert space, provided it is invariant with respect to subsystem permutations.

\section{Estimating the Size of a Sample}

Consider again a set of $m$ identical subsystems, with the same control capabilities as in the previous application: only the first $p$ are accessible via measurements of identical, nondegenerate observables $Q$ and feedback unitary control, while SSC can be reached on the whole network. We are now interested in estimating the number $m$ of subsystems in the quantum network.

For this, we will first prepare the network in a state $\rho^{\prime}$ that has support in a subspace which is orthogonal to some "marker" eigenstate $|\psi\rangle$ of $Q$, such that $\operatorname{Tr}\left[\rho^{\prime}(|\psi\rangle\langle\psi|)^{(j)}\right]=0$ for all $j$. Such a state can be asymptotically reached with an easy adaptation of the protocol described in the last section.

In order to estimate the size of the sample, we next apply the following procedure.

Step 1) Perform measurements of $Q$ on each of the $p$ probe subsystems, and use fast unitary control on each of them in order to prepare them all in the marker eigenstate $|\psi\rangle$ of $Q$.

Step 2) Let the network evolve with gossip to SSC.

Step 3) Perform again measurements of $Q$ on the $p$ probe subsystems, recording how many times $|\psi\rangle$ is obtained.

Step 1 prepares the network into a state

$$
|\psi\rangle\langle\psi|\otimes \ldots \otimes| \psi\rangle\langle\psi| \otimes \rho_{\backslash p}
$$

where $\rho_{\backslash p}$ is an unknown state on $m-p$ subsystems (with $m$ unknown), but still satisfying $\operatorname{Tr}\left[\rho_{\backslash p}(|\psi\rangle\langle\psi|)^{(j)}\right]=0$ for all $j>p$. As shown in Section V-A, the statistics of measuring $Q$ on the $p$ probe subsystems after Step 2, equals the statistics of measuring $Q$ before Step 2 on $p$ uniformly randomly selected subsystems. In the latter case, whenever one of the first $p$ subsystems was selected we would get outcome $|\psi\rangle$, while whenever a subsystem $j>p$ is selected we would certainly not get $|\psi\rangle$. The random variable $K$ counting the number $k$ of times $|\psi\rangle$ is detected in Step 3 therefore follows a hypergeometric distribution,

$$
K=k \text { with probability }\left(\begin{array}{l}
p \\
k
\end{array}\right)\left(\begin{array}{c}
m-p \\
p-k
\end{array}\right) /\left(\begin{array}{l}
m \\
p
\end{array}\right)
$$

where $\left(\begin{array}{l}b \\ a\end{array}\right)=b ! /(a !(b-a) !)$. We thus have

$$
\mathbb{E}[K]=p^{2} / m, \quad \operatorname{Var}(K)=\mathbb{E} \mid !\left[(K-\mathbb{E}(K))^{2}\right]=\frac{p^{2}(m-p)^{2}}{m^{2}(m-1)} .
$$

Then, the estimator can be chosen to be $\hat{m}=p^{2} / \hat{K}$, where $\hat{K}$ is the sampled value of $K$. It is then easier to study the statistical properties of $\hat{m}^{-1}$, being just a rescaling of the measured $\hat{K}$. The relative error $\left(\hat{m}^{-1}-m^{-1}\right) / m^{-1}$ of $\hat{m}^{-1}$ then has mean zero, i.e., it is an unbiased estimator. We can then compute its variance

$$
\begin{aligned}
\mathbb{E}\left[\left(\frac{\hat{m}^{-1}-m^{-1}}{m^{-1}}\right)^{2}\right] & =\mathbb{E}\left[m^{2}\left(\frac{\hat{K}}{p^{2}}-\frac{1}{m}\right)^{2}\right]=\frac{m^{2}}{p^{4}} \operatorname{Var}(K) \\
& =\frac{m^{2}}{p^{4}} \cdot \frac{p^{2}(m-p)^{2}}{m^{2}(m-1)}=\frac{(m-p)^{2}}{p^{2}(m-1)}
\end{aligned}
$$


This shows that if we pick $p=\alpha \cdot m$ to be a fixed yet unknown fraction of the total population, when the population increases the relative accuracy of $\hat{m}^{-1}$ improves since the above variance goes to zero as $1 / m$. Then for the limit of large $m$, we can conclude that the variance of $\hat{m}$ also goes to zero as $1 / \mathrm{m}$.

\section{Conclusion and Research Directions}

In this paper, we develop a general framework for posing and studying consensus problems in the quantum domain, and illustrate how it could be used for distributed control and estimation problems. In particular, we build on the statistical property of the states with respect to local observables and their symmetry with respect to permutation operations to derive four different generalizations of a consensus state to quantum systemsnamely $\sigma$-expectation consensus, reduced state consensus, symmetric state consensus, and single $\sigma$-measurement consensus - and establish their hierarchy. We highlight at each step the symmetry considerations underlying the results, making explicit connection with the usual multi-agent consensus problem. These ideas can be directly translated in order to obtain a "consensus on probabilities" framework for classical systems. With respect to the existing work on non-commutative consensus [17], our approach follows the analogy with the classical setting as closely as possible, maintaining an operational viewpoint and working with a multipartite system (a quantum network). We propose and analyze a quantum gossip-type algorithm that asymptotically prepares symmetricstate consensus states while preserving the expectation of any permutation invariant observable.

A number of questions remain open. Among these, we believe that it would be particularly interesting to further explore the link between single $\sigma$-measurement consensus states and entangled states [21], and to determine if, and under which conditions, it is possible to achieve this type of consensus with a distributed algorithm. This could potentially lead to a class of algorithms that prepare entangled states in a robust and distributed way. Another interesting point is to assess the potential of continuous-time dynamics for consensus: a first dissipative proposal has been presented in [30], but we believe it would be worth exploring also time-averages of Hamiltonian dynamics, which could lead to connections with physically relevant many-body dynamics.

Lastly, let us remark that in this paper we proposed a quantum algorithm in which the gossip-type interactions are selected in a classical way. The advantage of a fully quantum implementation, along with its potential speedup by using quantum random walks and their fast mixing properties [31], [32], is definitely worth further investigation.

\section{APPENDIX}

\section{A. Description of Quantum Systems and Notations}

1) Quantum Systems Basics: This paper considers networks of quantum systems. The mathematical description of a single quantum system starts by considering a complex Hilbert space $\mathcal{H}$. In order to be consistent with most of the literature on quantum control and quantum information, we shall employ Dirac's notation, where $|\psi\rangle$ denotes an element of $\mathcal{H}$ (called a ket), while $\langle\psi|=| \psi\rangle^{\dagger}$ is used for its dual (a bra), and $\langle\psi \mid \varphi\rangle$ for the associated inner product. We denote the set of linear operators on $\mathcal{H}$ by $\mathfrak{B}(\mathcal{H})$. The adjoint operator $X^{\dagger} \in \mathfrak{B}(\mathcal{H})$ of an operator $X \in \mathfrak{B}(\mathcal{H})$ is the unique operator that satisfies $(X|\psi\rangle)^{\dagger}|\chi\rangle=\langle\psi|\left(X^{\dagger}|\chi\rangle\right)$ for all $|\psi\rangle,|\chi\rangle \in \mathcal{H}$. We then denote $\mathfrak{H}(\mathcal{H})$ the subset of $\mathfrak{B}(\mathcal{H})$ of self-adjoint operators, and $\mathfrak{U}(\mathcal{H}) \subset \mathfrak{B}(\mathcal{H})$ the subset of unitary operators. The natural inner product in $\mathfrak{B}(\mathcal{H})$ is the Hilbert-Schmidt product $\langle X, Y\rangle=$ $\operatorname{Tr}\left(X^{\dagger} Y\right)$, where $\operatorname{Tr}$ is the usual trace functional (which is canonically defined in a finite dimensional setting). We denote by $I$ the identity operator.

While most of our results can be straightforwardly extended to a finite network of infinite-dimensional subsystems, for the sake of simplicity we restrict the presentation to finitedimensional systems. That is we shall assume $\mathcal{H} \approx \mathbb{C}^{n}$ for some $n$. Working in a finite dimensional setting, we can conveniently fix a basis in $\mathcal{H}$ and represent vectors and operators as complex matrices of suitable dimensions:

1) $|\psi\rangle \in \mathcal{H} \simeq \mathbb{C}^{n}$ are represented by column vectors;

2) $\langle\phi| \in \mathcal{H}^{\dagger} \simeq \mathbb{C}^{n}$ are row vectors;

3) $X \in \mathfrak{B}(\mathcal{H}) \simeq \mathbb{C}^{n \times n}$ are $n \times n$ complex matrices.

The adjoint $X^{\dagger}$ is then the transpose conjugate of $X$, and self-adjoint and unitary properties carry over to the associated matrices (Hermitian and unitary, respectively). Readers that are not familiar with quantum theory can keep in mind this representation and follow this paper in linear algebraic terms.

In statistical quantum theory, the state of a quantum system is represented by a density operator $\rho$, that is any self-adjoint, positive semi-definite operator with trace one. We denote the convex set of these operators (the state space) by $\mathfrak{D}(\mathcal{H})$. The extreme points of this set, namely the rank-one operators $\rho=$ $|\psi\rangle\langle\psi|$ with $|\psi\rangle \in \mathcal{H}$ and $\langle\psi \mid \psi\rangle=1$, are called pure states.

A projective (or von Neumann) observation, or measurement, of a quantum system is characterized by a so-called observable, that is a self-adjoint operator $\sigma \in \mathfrak{H}(\mathcal{H})$, see, e.g., [20]. Its spectral decomposition $\sigma=\sum_{j=1}^{d} s_{j} \Pi_{j}$, with $d \leq n$ distinct eigenvalues $\left\{s_{j}\right\}$ and projectors onto associated eigenspaces $\left\{\Pi_{j}\right\}$, governs the stochastic outcome of the measurement and the possibly modified state of the system after measurement: having state $\rho$ before the measurement, the latter's outcome will be $s_{j}$ with probability $\mathbb{P}_{\rho}\left(s_{j}\right)=\operatorname{Tr}\left(\Pi_{j} \rho\right)=: p_{j}$; and if outcome $s_{j}$ is obtained, then the state after the measurement is $\left.\rho\right|_{j}=\Pi_{j} \rho \Pi_{j} / p_{j}$. The probability to observe $s_{k}^{\prime}$ in a subsequent measurement of $\sigma^{\prime}=\sum_{k=1}^{d^{\prime}} s_{k}^{\prime} \Pi_{k}^{\prime}$, with eigenvalues $\left\{s_{k}^{\prime}\right\}$ and projectors onto associated eigenspaces $\left\{\Pi_{k}^{\prime}\right\}$ that do not necessarily commute with the $\Pi_{j}$, is then

$$
\mathbb{P}_{\left.\rho\right|_{j}}\left(\Pi_{k}^{\prime}\right)=\operatorname{Tr}\left(\Pi_{k}^{\prime} \Pi_{j} \rho \Pi_{j}\right) / p_{j} .
$$

It follows that the probability of observing the ordered sequence of two events first $s_{j}$, then $s_{k}^{\prime}$, given the initial $\rho$, is

$$
\mathbb{P}_{\rho}\left(\Pi_{j}, \Pi_{k}^{\prime}\right)=\operatorname{Tr}\left(\Pi_{k}^{\prime} \Pi_{j} \rho \Pi_{j}\right) .
$$

If $\Pi_{j}$ and $\Pi_{k}^{\prime}$ do not commute, a different ordering in a sequence of measurements can change the resulting probability. If $\Pi_{j}$ and $\Pi_{k}^{\prime}$ do commute, and only then, the joint probability of observing $s_{j}, s_{k}$ is independent of the measurement order for all $\rho$, and simplifies to

$$
\mathbb{P}_{\rho}\left(\Pi_{j}, \Pi_{k}^{\prime}\right)=\operatorname{Tr}\left(\Pi_{k}^{\prime} \Pi_{j} \rho\right) .
$$

2) Two-Level Quantum Systems as Qubits: A qubit is a (generic, abstract) quantum system associated to a twodimensional Hilbert space $\mathcal{H} \sim \mathbb{C}^{2}$; a standard basis for the 
latter is conventionally given by two vectors denoted $|0\rangle \sim$ $[1,0]^{T}$ and $|1\rangle \sim[0,1]^{T}$.

The traceless unitary hermitian Pauli operators $\sigma_{x}, \sigma_{y}, \sigma_{z}$ and the identity operator $I$ together form an orthonormal basis for all hermitians operators on $\mathcal{H}$. Explicitly, $\sigma_{x}=|1\rangle\langle 0|+$ $|0\rangle\left\langle 1\left|, \sigma_{y}=i\right| 1\right\rangle\langle 0|-i| 0\rangle\left\langle 1\left|, \sigma_{z}=\right| 0\right\rangle\langle 0|-| 1\rangle\langle 1|$. With the standard basis, these are associated to the matrices

$$
\sigma_{x}=\left[\begin{array}{ll}
0 & 1 \\
1 & 0
\end{array}\right], \quad \sigma_{y}=\left[\begin{array}{cc}
0 & -i \\
i & 0
\end{array}\right], \quad \sigma_{z}=\left[\begin{array}{cc}
1 & 0 \\
0 & -1
\end{array}\right] .
$$

These all have eigenvalues $1,-1$.

We briefly illustrate quantum projective measurement for this example.

Assume for instance that the initial state is $\rho=(1 / 3)|0\rangle\langle 0|+$ $(2 / 3)|1\rangle\langle 1|$ and we perform a measurement of $\sigma_{z}=|0\rangle\langle 0|-$ $|1\rangle\langle 1|=s_{1} \Pi_{1}+s_{2} \Pi_{2}$, where $\Pi_{1}=|0\rangle\langle 0|$ and $\Pi_{2}=|1\rangle\langle 1|$. Then we get outcome $s_{1}=1$ with probability $\operatorname{Tr}\left(\rho \Pi_{1}\right)=$ $1 / 3$, and if that is recorded, we update the state to $\Pi_{1} \rho \Pi_{1} /$ $\operatorname{Tr}\left(\Pi_{1} \rho \Pi_{1}\right)=|0\rangle\langle 0|$. Outcome $s_{2}=-1$ will appear with probability $2 / 3$, and in that case we shall transform the state to $\Pi_{2} \rho \Pi_{2} / \operatorname{Tr}\left(\Pi_{2} \rho \Pi_{2}\right)=|1\rangle\langle 1|$. Consider now the same initial state $\rho$ but we perform a measurement of $\sigma_{x}$. Then similar calculations yield that we get outcome $s_{1}=1$ with probability $\operatorname{Tr}\left(\rho \Pi_{1}\right)=1 / 2$, with now $\Pi_{1}=(1 / 2)(|0\rangle+|1\rangle)(\langle 0|+\langle 1|)$, while the post-measurement state gets updated to $\Pi_{1}$; and with probability $\operatorname{Tr}\left(\rho \Pi_{2}\right)=1 / 2$ we get outcome $s_{2}=-1$, with $\Pi_{2}=(1 / 2)(|0\rangle-|1\rangle)(\langle 0|-\langle 1|)$, and update the state to $\Pi_{2}$. If we perform a measurement of $I$ instead, then we always get the unique result 1 and the state $\rho$ does not change. For systems on higher-dimensional Hilbert spaces, measurements associated to degenerate operators can project the state to a subspace of dimension $>1$, leading after measurement to a modified state which depends on the initial state. This is always the case when carrying out a measurement on one part of a multipartite quantum system.

3) Multipartite Systems and Partial Trace: For simplicity, we present the interaction of two quantum systems; the case of $n>2$ systems is easily obtained by iteration. If two quantum systems, with associated Hilbert spaces $\mathcal{H}_{1}$ and $\mathcal{H}_{2}$, respectively, are taken together to form a larger bipartite quantum system, the Hilbert space $\mathcal{H}_{1,2}$ associated to the composite quantum system is the tensor product of the individual quantum subsystem Hilbert spaces, $\mathcal{H}_{1} \otimes \mathcal{H}_{2}$.

Let $\left\{\left|\psi_{k}\right\rangle\right\}_{k=1}^{n_{1}}$ and $\left\{\left|\phi_{l}\right\rangle\right\}_{l=1}^{n_{2}}$ be orthonormal bases for $\mathcal{H}_{1}$ and $\mathcal{H}_{2}$, respectively, then an orthonormal basis for $\mathcal{H}_{1,2}$ is

$$
\left\{\left|\psi_{k}\right\rangle \otimes\left|\phi_{l}\right\rangle\right\}_{k, l=1}^{n_{1}, n_{2}}
$$

from which we get that $\operatorname{dim}\left(\mathcal{H}_{1,2}\right)=\operatorname{dim}\left(\mathcal{H}_{1}\right) \operatorname{dim}\left(\mathcal{H}_{2}\right)=$ $n_{1} n_{2}$. We use the short notation $|\psi, \phi\rangle:=|\psi\rangle \otimes|\phi\rangle$ for any $|\psi\rangle \in \mathcal{H}_{1}$ and $|\phi\rangle \in \mathcal{H}_{2}$. The composite Hilbert space is naturally endowed with the inner-product $\left\langle u_{1}, u_{2} \mid v_{1}, v_{2}\right\rangle:=$ $\left\langle u_{1} \mid v_{1}\right\rangle\left\langle u_{2} \mid v_{2}\right\rangle$. A representation and basis for operators in $\mathfrak{B}\left(\mathcal{H}_{1,2}\right)$ is derived from its vector counterpart in the standard way. In particular, given two operators $X_{1} \in \mathfrak{B}\left(\mathcal{H}_{1}\right)$ and $X_{2} \in$ $\mathfrak{B}\left(\mathcal{H}_{2}\right)$, one can define $X_{1} \otimes X_{2} \in \mathfrak{B}\left(\mathcal{H}_{1,2}\right)$ as the linear operator such that $\forall\left|u_{1}\right\rangle \in \mathcal{H}_{1},\left|u_{2}\right\rangle \in \mathcal{H}_{2}$ :

$$
X_{1} \otimes X_{2}\left(\left|u_{1}\right\rangle \otimes\left|u_{2}\right\rangle\right)=X_{1}\left|u_{1}\right\rangle \otimes X_{2}\left|u_{2}\right\rangle \text {. }
$$

If two operators are in the form $X_{1} \otimes \mathcal{I}_{2}$ and $\mathcal{I}_{1} \otimes X_{2}$, i.e., they act nontrivially only on different parts of the multipartite sys- tem, then they commute for any $X_{1}$ and $X_{2}$. It is worth noting that in matrix representation, the tensor product corresponds to the Kronecker product.

The partial trace over $\mathcal{H}_{1}$ is the unique linear map

$$
\operatorname{Tr}_{\mathcal{H}_{1}}: \mathfrak{B}\left(\mathcal{H}_{1} \otimes \mathcal{H}_{2}\right) \longrightarrow \mathfrak{B}\left(\mathcal{H}_{2}\right)
$$

such that, for any $X_{1,2} \in \mathfrak{B}\left(\mathcal{H}_{1,2}\right)$ and any $X_{2} \in \mathfrak{B}\left(\mathcal{H}_{2}\right)$,

$$
\operatorname{Tr}\left[\operatorname{Tr}_{\mathcal{H}_{1}}\left[X_{1,2}\right] X_{2}\right]=\operatorname{Tr}\left[X_{1,2}\left(\mathcal{I}_{1} \otimes X_{2}\right)\right]
$$

If $\left\{\left|\psi_{k}\right\rangle\right\}_{k=1}^{n_{1}}$ and $\left\{\left|\phi_{l}\right\rangle\right\}_{l=1}^{n_{2}}$ are orthonormal bases for $\mathcal{H}_{1}$ and $\mathcal{H}_{2}$, respectively, the partial trace over $\mathcal{H}_{1}$ can be written as

$$
\operatorname{Tr}_{\mathcal{H}_{1}}\left[X_{1,2}\right]=\sum_{k=1}^{n_{1}} \sum_{l, i=1}^{n_{2}}\left\langle\psi_{k} \otimes \phi_{l}\left|X_{1,2}\right| \psi_{k} \otimes \phi_{i}\right\rangle\left|\phi_{l}\right\rangle\left\langle\phi_{i}\right| .
$$

The partial trace over $\mathcal{H}_{2}$ writes in a similar fashion.

\section{B. Proofs of Results in Section II-B}

Proof of Proposition 1: By considering an hermitian basis for $\mathfrak{B}(\mathcal{H})$, it is clear that a state is RSC if it is $\sigma \mathrm{EC}$ for all $\sigma \in \mathfrak{B}(\mathcal{H})$.

If $\bar{\rho}_{k}$ is a pure state for each $k$, then necessarily $\rho=$ $|\psi\rangle\langle\psi|$ with $|\psi\rangle=\left|\psi_{1}\right\rangle \otimes\left|\psi_{2}\right\rangle \otimes \ldots \otimes\left|\psi_{m}\right\rangle$ for some $\left|\psi_{k}\right\rangle$, $k=1,2, \ldots, m$. If in addition we require $\mathrm{RSC}$, then we need $\left|\psi_{k}\right\rangle\left\langle\psi_{k}\left|=\bar{\rho}_{k}=\bar{\rho}_{j}=\right| \psi_{j}\right\rangle\left\langle\psi_{j}\right|$ for all $j, k$; thus, $\left|\psi_{k}\right\rangle=\left|\psi_{j}\right\rangle$ up to an irrelevant phase factor for all $j, k$ and particle permutation indeed leaves $|\psi\rangle$ invariant.

Proof of Theorem 2: Note that the properties $\operatorname{Tr}\left(\Pi_{\text {sym }} \rho\right)=$ 1 and $\Pi_{\text {sym }} \rho \Pi_{\text {sym }}=\Pi_{\text {sym }} \rho=\rho$ are equivalent because $\Pi_{\text {sym }}$ is an orthonormal projector and $\rho$ is self-adjoint positive semidefinite with unit trace. Assume (5) to hold. Along with the identities $\Pi_{j}^{(k)} \Pi_{\mathrm{sym}}=\Pi_{j}^{(k)} \Pi_{j}^{\otimes m}=\Pi_{j}^{\otimes m}$, this gives

$$
\begin{aligned}
\operatorname{Tr}\left(\Pi_{j}^{(\ell)} \Pi_{j}^{(k)} \rho\right) & =\operatorname{Tr}\left(\Pi_{j}^{(\ell)} \Pi_{j}^{(k)} \Pi_{\text {sym }} \rho\right)=\operatorname{Tr}\left(\Pi_{j}^{(\ell)} \Pi_{j}^{\otimes m} \rho\right) \\
& =\operatorname{Tr}\left(\Pi_{j}^{(\ell)} \Pi_{\text {sym }} \rho\right)=\operatorname{Tr}\left(\Pi_{j}^{(\ell)} \rho\right)
\end{aligned}
$$

for all $j, k, \ell$. Hence, the $\sigma \mathrm{SMC}$ definition (4) indeed holds.

On the other hand, suppose that (5) does not hold. This means that $\operatorname{Tr}\left(\left(I-\Pi_{\text {sym }}\right) \rho\right)>0$. We want to show that this implies

$$
\operatorname{Tr}\left(\Pi_{j}^{(k)} \Pi_{j}^{(\ell)} \rho\right) \neq \operatorname{Tr}\left(\Pi_{j}^{(\ell)} \rho\right)
$$

for some $j, k, \ell$. Let us write

$$
I-\Pi_{\mathrm{sym}}=\sum_{\substack{j_{1}, \ldots, j_{m} \in\{1,2, \ldots, d\} \\ \text { except }\left\{j_{1}=\ldots=j_{m}\right\}}} \Pi_{j_{1}} \otimes \ldots \otimes \Pi_{j_{m}} .
$$

Since $\operatorname{Tr}\left(\left(I-\Pi_{\text {sym }}\right) \rho\right)>0$ implies that $\operatorname{Tr}\left(\Pi_{j_{1}} \otimes \ldots \otimes\right.$ $\left.\Pi_{j_{m}} \rho\right)>0$ for at least one of the terms in the above sum, let us take one such term, denote the corresponding indices as $\left\{\bar{j}_{s}\right\}$ and denote by $k, \ell$ two subsystems such that $\bar{j}_{k} \neq \bar{j}_{\ell}$ in that term. Now writing $\Pi_{\bar{j}_{1}} \otimes \ldots \otimes \Pi_{\bar{j}_{m}}=\Pi_{\bar{j}_{1}}^{(1)} \Pi_{\bar{j}_{2}}^{(2)} \ldots \Pi_{\bar{j}_{m}}^{(m)}$, where all factors commute, we have

$$
\operatorname{Tr}\left(\Pi_{\bar{j}_{k}}^{(k)} \Pi_{\bar{j}_{\ell}}^{(\ell)} \rho\right) \geq \operatorname{Tr}\left(\Pi_{\bar{j}_{1}}^{(1)} \Pi_{\bar{j}_{2}}^{(2)} \ldots \Pi_{\bar{j}_{m}}^{(m)} \rho\right)>0 .
$$

By mutual orthogonality of $\left\{\Pi_{\bar{j}_{a}}^{(k)}: a=1,2, \ldots, m\right\}$ for fixed $k$, and knowing that the trace of an operator cannot increase 
under multiplication by a projection operator, we thus get

$$
\begin{aligned}
\operatorname{Tr}\left(\Pi_{\bar{j}_{\ell}}^{(k)} \Pi_{\bar{j}_{\ell}}^{(\ell)} \rho\right) & \leq \operatorname{Tr}\left(\left(1-\Pi_{\bar{j}_{k}}^{(k)}\right) \Pi_{\bar{j}_{\ell}}^{(\ell)} \rho\right) \\
& =\operatorname{Tr}\left(\Pi_{\bar{j}_{\ell}}^{(\ell)} \rho\right)-\operatorname{Tr}\left(\Pi_{\bar{j}_{k}}^{(k)} \Pi_{\bar{j}_{\ell}}^{(\ell)} \rho\right) \\
& \leq \operatorname{Tr}\left(\Pi_{\bar{j}_{\ell}}^{(\ell)} \rho\right)-\operatorname{Tr}\left(\Pi_{\bar{j}_{1}}^{(1)} \Pi_{\bar{j}_{2}}^{(2)} \ldots \Pi_{\bar{j}_{m}}^{(m)} \rho\right) \\
& <\operatorname{Tr}\left(\Pi_{\bar{j}_{\ell}}^{(\ell)} \rho\right) .
\end{aligned}
$$

For (a), we have since (4) holds for all $k, \ell$ :

$$
\operatorname{Tr}\left(\Pi_{j}^{(k)} \rho\right)=\operatorname{Tr}\left(\Pi_{j}^{(k)} \Pi_{j}^{(\ell)} \rho\right)=\operatorname{Tr}\left(\Pi_{j}^{(\ell)} \rho\right) .
$$

By linearity, we thus have

$$
\operatorname{Tr}\left(\sigma^{(k)} \rho\right)=\sum_{j=1}^{d} s_{j} \operatorname{Tr}\left(\Pi_{j}^{(k)} \rho\right)=\sum_{j=1}^{d} s_{j} \operatorname{Tr}\left(\Pi_{j}^{(\ell)} \rho\right)=\operatorname{Tr}\left(\sigma^{(\ell)} \rho\right) .
$$

A counterexample for the converse is state $\rho^{A}$ in Example 1 .

Counterexamples for the converse of (b) and (c) are, respectively, states $\rho^{B}$ and $\rho^{C}$ in Example 1. For the direct statements, given Proposition 1, we know that if (c) is true, then (b) must be true as well. Let us then focus on (c). Take the representation of $\rho$ in the basis associated to $\sigma=\sum_{j=1}^{n} s_{j}|j\rangle\langle j|$, where thus $|j\rangle\langle j|=\Pi_{j}$, that reads

$$
\rho=\sum_{\substack{j_{1}, j_{2}, \ldots j_{m} \in D, k_{1}, k_{2}, \ldots k_{m} \in D}} r_{\substack{j_{1}, j_{2}, \ldots j_{m} \\ k_{1}, k_{2}, \ldots k_{m}}}\left|j_{1}, j_{2}, \ldots, j_{m}\right\rangle\left\langle k_{1}, k_{2}, \ldots, k_{m}\right|
$$

with $D=\{1,2, \ldots, n\}$. From Proposition 2, the condition for $\sigma \mathrm{SMC}$ writes

$$
\sum_{k, j \in D}(|k\rangle\langle k|)^{\otimes m} \rho(|j\rangle\langle j|)^{\otimes m}=\rho
$$

so (41) must reduce to

$$
\rho=\sum_{k, j \in D} p_{k j}|k k \ldots k\rangle\langle j j \ldots j|
$$

for some $p_{k j} \in \mathbb{C}$. It is straightforward to see that a $\rho$ of this form satisfies SSC, since any element in the sum is invariant w.r.t. subsystem permutations. Regarding point (e), the definition of $\sigma$ SMC involves $\operatorname{Tr}\left(\Pi_{j}^{(k)} \Pi_{j}^{(\ell)} \rho\right)$, which takes the partial trace over the state of all subsystems except the pair $\{k, \ell\}$. So we can effectively discard all but two subsystems, and show without loss of generality that it is impossible to make $\sigma \mathrm{SMC}$ hold for all $\sigma$ on two subsystems $k=1, \ell=2$. In Proposition 2, we say that $\sigma \mathrm{SMC}$ for a particular $\sigma$ requires $\Pi_{\text {sym }} \rho=\rho$ with $\Pi_{\text {sym }}=\sum_{j=1}^{d} \Pi_{j}^{\otimes m}$, and $\left\{\Pi_{j}\right\}$ the spectral projectors associated to $\sigma$. So if $\sigma$ SMC has to hold for both $\sigma$ and $\sigma^{\prime}$, we must have in particular

$$
\Pi_{\text {sym }} \Pi_{\text {sym }}^{\prime} \Pi_{\text {sym }} \rho=\rho
$$

where $\Pi_{\mathrm{sym}}^{\prime}$ is associated to $\sigma^{\prime}$. Since $H:=\Pi_{\mathrm{sym}} \Pi_{\mathrm{sym}}^{\prime} \Pi_{\mathrm{sym}}$ and $\rho$ both are self-adjoint positive semidefinite, the only way to have $H \rho=\rho \neq 0$ is if $H$ has at least one eigenvalue $\geq 1$. Now take in particular $\sigma=\sum_{k=1}^{n} k\left|x_{k}\right\rangle\left\langle x_{k}\right|$ and $\sigma^{\prime}=$ $\sum_{k=1}^{n} k\left|p_{k}\right\rangle\left\langle p_{k}\right|$, with $\quad p_{k}=(1 / \sqrt{n}) \sum_{j=0}^{n-1} e^{j k 2 \pi i / n}\left|x_{j+1}\right\rangle$ (thus the $\left|p_{k}\right\rangle$-basis is related to the $\left|x_{k}\right\rangle$-basis by Fourier transform). A few computations show that $H$ then has all eigenvalues $<1$, except for $n=2$ that is the case of two qbits.
For the latter particular case, one can prove the property by showing, e.g., that there is no state which would satisfy $\sigma$ SMC for all $\sigma \in\left\{\sigma_{x}, \sigma_{y}, \sigma_{z}\right\}$.

\section{On Detecting Quantum Consensus}

In the quantum setting, there exists no state $\rho$ for which all measurement outcomes are deterministically defined. Even a maximal information state, i.e., $\rho=|\psi\rangle\langle\psi|$ a rank one projector, leads to probabilistic outcomes for all observables of which $|\psi\rangle$ is not an eigenstate. As we are thus compelled to use probabilistic notions, consensus can only be inferred from stochastic measurement records, and checking different types of consensus requires different types of measurement statistics.

The $\sigma \mathrm{EC}$, requiring only equal expectations for a particular observable $\sigma$ on the different subsystems, simply requires measurements of local $\sigma$-measurements results, but no correlations between measurement results on different subsystems are needed. Checking RSC requires statistics for a basis of observables for each subsystem; as for $\sigma \mathrm{EC}$, correlations between measurement results on different subsystems play no role. On the other hand, distinguishing SSC from RSC does require to inspect correlations between measurement outcomes at different subsystems.

Proposition 5: Except for the case of reduced pure states considered in Proposition 1, SSC can only be distinguished from RSC by inspecting correlations between measurement outcomes at different subsystems.

Proof: The statement builds on the standard fact that the statistics of a local observable $\sigma_{1} \otimes \sigma_{2} \otimes \ldots \otimes \sigma_{m}$ only depend on reduced states $\bar{\rho}_{1}, \bar{\rho}_{2}, \ldots, \bar{\rho}_{m}$. So repeated local measurements can, at their best, fully characterize the $\bar{\rho}_{k}$. Checking RSC, i.e., that these $\bar{\rho}_{k}$ are all equal, is thus straightforward. On the other hand, reduced states $\bar{\rho}_{k}$ are the best that can be extracted by local measurements in trying to distinguish RSC from SSC states. If $\bar{\rho}_{1}=\bar{\rho}_{2}=\ldots=: \bar{\rho}$ have rank one, we have the special case that is always SSC. If instead $\bar{\rho}$ has rank at least 2 , we can write it as $\bar{\rho}=p_{1} R_{1}+p_{2} R_{2}$ where $R_{1}, R_{2} \in \mathfrak{B}(\mathcal{H})$, $p_{1}, p_{2}$ are positive scalars, $R_{2}$ is positive semidefinite, and $R_{1}$ is a projector on a 2-D subspace $\mathcal{V}_{2}$. Consider $R_{1}=\left|e_{1}\right\rangle\left\langle e_{1}\right|+$ $\left|e_{2}\right\rangle\left\langle e_{2}|=| f_{1}\right\rangle\left\langle\left|f_{1}+\right| f_{2}\right\rangle\left\langle f_{2}\right|$, where $\left|e_{1}\right\rangle,\left|e_{2}\right\rangle$ and $\left|f_{1}\right\rangle,\left|f_{2}\right\rangle$ are two orthonormal bases for $\mathcal{V}_{2}$ with $\left\langle e_{1} \mid f_{1}\right\rangle \notin\{0,1\}$. Then $\bar{\rho}$ could equally well reflect the state

$$
\rho=\bar{\rho}^{\otimes m}
$$

which is SSC, or, e.g., a state of the form

$$
\begin{array}{r}
\rho=p_{2} R_{2}^{\otimes m}+p_{1}\left(\left|e_{1}\right\rangle\left|f_{1}\right\rangle+\left|e_{2}\right\rangle\left|f_{2}\right\rangle\right)\left(\left|e_{1}\right\rangle\left|f_{1}\right\rangle+\left|e_{2}\right\rangle\left|f_{2}\right\rangle\right)^{\dagger} \\
\otimes R_{1}^{\otimes(m-2)}
\end{array}
$$

where the first two subsystems are entangled. This state is not SSC, even for $m=2$. Thus, the local knowledge of $\bar{\rho}$ does not allow to distinguish if the state is SSC or not.

For instance, considering the state $\rho^{B}$ of Example 1, measurements of $\sigma_{z}$ on the three subsystems would quickly show that the results on subsystems 2 and 3 are always perfectly correlated, and show no correlation at all with the results on the first subsystem. This difference in correlations rules out $\rho^{B}$ as a candidate for SSC.

The definition of $\sigma \mathrm{SMC}$ is all about correlations between measurement outcomes at different subsystems: the latter must 
be fully correlated for a particular observable $\sigma$. Positively detecting states in SSC but not in SMC, however, appears to be less obvious (except through full state tomography).

\section{REFERENCES}

[1] J. N. Tsitsiklis, "Problems in decentralized decision making and computation," Ph.D. dissertation, Mass. Inst. of Technol., Cambridge, MA, USA, 1984.

[2] R. Olfati-Saber, J. Fax, and R. Murray, "Consensus and cooperation in networked multi-agent systems," Proc. IEEE, vol. 95, no. 1, pp. 215-233, Jan. 2007.

[3] E. Knill and R. Laflamme, "Theory of quantum error-correcting codes," Phys. Rev. A, vol. 55, no. 2, pp. 900-911, Feb. 1997.

[4] R. Blume-Kohout, H. K. Ng, D. Poulin, and L. Viola, "Information preserving structures: A general framework for quantum zero-error information," Preprint, vol. arxiv, p. 1006.1358v1, 2010.

[5] L. Viola and E. Knill, "Random decoupling schemes for quantum dynamical control and error suppression," Phys. Rev. Lett., vol. 94, p. 060502, 2005.

[6] F. Ticozzi and L. Viola, "Quantum information encooding, protection and correction via trace-norm isometries," Phys. Rev. A, vol. 81, no. 3, p. 032313, 2010.

[7] S. Lloyd and L. Viola, "Engineering quantum dynamics," Phys. Rev. A, vol. 65, pp. 010101:1-010101:4, 2001.

[8] J. T. Barreiro, M. Muller, P. Schindler, D. Nigg, T. Monz, M. Chwalla, M. Hennrich, C. F. Roos, P. Zoller, and R. Blatt, "An open-system quantum simulator with trapped ions," Nature, vol. 470, pp. 486-491, 2011

[9] F. Verstraete, M. M. Wolf, and J. I. Cirac, "Quantum computation and quantum-state engineering driven by dissipation," Nature Phys., vol. 5, pp. 633-636, 2009.

[10] B. Kraus, S. Diehl, A. Micheli, A. Kantian, H. P. Büchler, and P. Zoller, "Preparation of entangled states by dissipative quantum markov processes," Phys. Rev. A, vol. 78, no. 4, p. 042307, 2008.

[11] F. Ticozzi and L. Viola, "Stabilizing entangled states with quasi-local quantum dynamical semigroups," Phil. Trans. R. Soc. A, vol. 370, no. 1979 , pp. 5259-5269, 2012.

[12] C. Altafini and F. Ticozzi, "Modeling and control of quantum systems: An introduction," IEEE Trans. Autom. Control, vol. 57, no. 8, pp. 1898-1917, Aug. 2012.

[13] H. M. Wiseman and G. J. Milburn, Quantum Measurement and Control. Cambridge, U.K.: Cambridge Univ. Press, 2009.

[14] J. Gough and M. R. James, "The series product and its application to quantum feedforward and feedback networks," IEEE Trans. Autom. Control, vol. 54, no. 11, pp. 2530-2544, Nov. 2009.

[15] R. F. Werner, "Quantum states with Einstein-Podolsky-Rosen correlations admitting a hidden-variable model," Phys. Rev. A, vol. 40, pp. 4277-4281, 1989.

[16] T. Eggeling and R. F. Werner, "Separability properties of tripartite states with $u \bigotimes u \bigotimes u$ symmetry," Phys. Rev. A, vol. 63, p. 042111, 2001.

[17] R. Sepulchre, A. Sarlette, and P. Rouchon, "Consensus in noncommutative spaces," in Proc. 49th IEEE Conf. Decision Control, 2010, pp. 6596-6601.

[18] H. Maassen, "Quantum probability applied to the damped harmonic oscillator," in Quantum Probability Communications, vol. XII, S. Attal and J. Lindsay, Eds. Singapore: World Scientific, 2003, pp. 23-58.

[19] L. Bouten, R. van Handel, and M. R. James, "An introduction to quantum filtering," SIAM J. Control Optim., vol. 46, no. 2, pp. 2199-2241, 2007.

[20] J. J. Sakurai, Modern Quantum Mechanics. New York, NY, USA Addison-Wesley, 1994.

[21] M. A. Nielsen and I. L. Chuang, Quantum Computation and Information. Cambridge, U.K.: Cambridge Univ. Press, 2002.

[22] K. Kraus, States, Effects, and Operations: Fundamental Notions of Quantaum Theory. Berlin, Germany: Springer-Verlag, 1983, ser. Lecture notes in Physics.

[23] C. B. Mendl and M. M. Wolf, "Unital quantum channels-Convex structure and revivals of birkhoff's theorem," Commun. Math. Phys., vol. 289, pp. 1057-1096, 2009.
[24] L. Moreau, "Stability of multi-agent systems with time-dependent communication links," IEEE Trans. Autom. Control, vol. 50, no. 2, pp. 169-182, Feb. 2005.

[25] F. Albertini and F. Ticozzi, "Discrete-time controllability for feedback quantum dynamics," Automatica, vol. 47, no. 11, pp. 2451-2456, 2011.

[26] S. Boyd, A. Ghosh, B. Prabhakar, and D. Shah, "Randomized gossip algorithms," IEEE Trans. Inf. Theory (Special Issue), vol. 52, no. 6, pp. 2508-2530, Jun. 2006.

[27] H. K. Khalil, Nonlinear Systems, 3rd ed. Upper Saddle River, NJ, USA: Prentice-Hall, 2002.

[28] R. Alicki and K. Lendi, Quantum Dynamical Semigroups and Applications. Berlin, Germany: Springer-Verlag, 1987.

[29] S. Bolognani and F. Ticozzi, "Engineering stable discrete-time quantum dynamics via a canonical QR decomposition," IEEE Trans. Autom. Control, vol. 55, no. 12, pp. 2721-2734, Dec. 2010.

[30] F. Ticozzi, L. Mazzarella, and A. Sarlette, "Symmetrization for quantum networks: A continuous-time approach," in Proc. MTNS'14, 2014

[31] J. Kempe, "Quantum random walks: An introductory overview," Contemp. Phys., vol. 44, no. 4, pp. 307-327, 2003.

[32] M. Szegedy, "Quantum speed-up of Markov chain based algorithms," in Proc. 45th Annu. IEEE Symp. Foundations Comput. Sci., 2004, pp. 32-41.

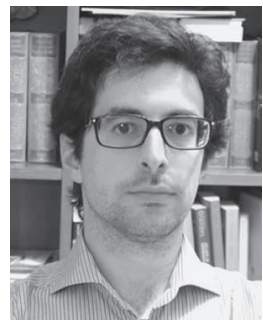

Luca Mazzarella received the B.S. degree in physics and the M.S. degree in theoretical physics form the University of Padova, Padua, Italy, in 2008 and 2010, respectively. He is currently pursuing the Ph.D. degree in the Department of Information Engineering, University of Padova.

His research interests include distributed and robust control of quantum networks and quantum transport in bio-inspired systems.

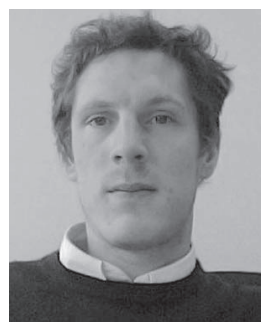

Alain Sarlette received the engineering degree in applied physics and the Ph.D. degree in applied sciences from the University of Liege, Liege, Belgium, in 2005 and 2009, respectively.

$\mathrm{He}$ has held visiting positions at Princeton University (2006) and Ecole des Mines de Paris (2009-2010). Since 2011, he has been a Lecturer at Ghent University, Ghent, Belgium. His research interests include nonlinear systems and geometric control approaches, coordination algorithms and distributed control, and quantum control.

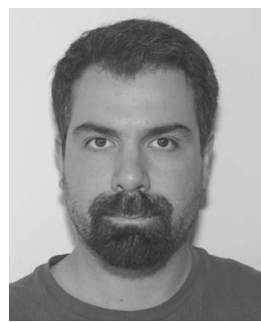

Francesco Ticozzi received the "Laurea" degree in management engineering and the Ph.D. degree in automatic control and operations research from the University of Padua, Padua, Italy, in 2002 and 2007, respectively.

Since February 2007, he has been with the Department of Information Engineering at the University of Padua, first as a Research Associate, and then as an Assistant Professor (Ricercatore). During 2005-2010, he held visiting appointments at the Physics and Astronomy Department, Dartmouth College, Hanover, NH, USA, where he has been an Adjunct Assistant Professor since July 2011. His research interests include modeling and control of quantum systems, protection of quantum information, quantum communications, and information-theoretic approaches to control systems. 